D.-C. Chang and B. Q. Li

Nagoya Math. J.

Vol. 147 (1997), 147-178

\title{
SOBOLEV AND LIPSCHITZ ESTIMATES FOR WEIGHTED BERGMAN PROJECTIONS
}

\author{
DER-CHEN CHANG AND BAO QIN LI ${ }^{1}$
}

Dedicated to the memory of Professor Yau-Cheun Wong

\begin{abstract}
Let $\Omega$ be a bounded, decoupled pseudo-convex domain of finite type in $\mathbb{C}^{n}$ with smooth boundary. In this paper, we generalize results of Bonami-Grellier $[\mathrm{BG}]$ and Bonami-Chang-Grellier [BCG] to study weighted Bergman projections for weights which are a power of the distance to the boundary. We define a class of operators of Bergman type for which we develop a functional calculus. Then we may obtain Sobolev and Lipschitz estimates, both of isotropic and anisotropic type, for these projections.
\end{abstract}

\section{$\S 1$. Introduction}

Let $\Omega \subset \mathbb{C}^{n}$ be a bounded, smooth pseudo-convex domain. Then, $\Omega$ is said to be decoupled of finite type near $\zeta \in \partial \Omega$ if there exists a holomorphic coordinate system $\left(z_{1}, \ldots, z_{n}\right)$ mapping $\zeta$ onto 0 and a neighborhood $U_{\zeta}$ of $\zeta$ onto a neighborhood $U$ of 0 and smooth, sub-harmonic but not harmonic functions $\left\{f_{j}\right\}_{\{j=1, \ldots, n-1\}}, f_{j}: \mathbb{C} \rightarrow \mathbb{R}$ with $f_{j}(0)=0$, and each $f_{j}$ vanishing to finite order at 0 , such that

$$
\left\{z \in U: \rho(z)=2 \operatorname{Im}\left(z_{n}\right)-\sum_{j=1}^{n-1} f_{j}\left(z_{j}\right)>0\right\} \simeq \Omega \cap U_{\zeta} .
$$

Let us denote by $m_{j}(\zeta)$ the order of vanishing of $f_{j}$ at 0 .

Notice that the finiteness condition here is equivalent to finite type in the case of real analytic pseudo-convex hypersurface $z \subset \mathbb{C}^{n}$ since the Levi form of $\Omega$ is diagonalizable (see Kohn [K1], [K2]).

Let $\zeta \in \partial \Omega$. We denote by

$$
\left(m_{1}, \ldots, m_{n-1}\right)=\max _{\zeta \in \partial \Omega}\left(m_{1}(\zeta), \ldots, m_{n-1}(\zeta)\right) .
$$

Received August 1, 1995.

${ }^{1}$ The research is partially supported by a grant from the National Science Foundation. 
As usual, the domain $\Omega$ is called of finite type $m$ with $m=\max \left\{m_{1}, \ldots\right.$, $\left.m_{n-1}\right\}$ if $m<\infty$. Let $f_{j}$ be the functions given by (1.1) and denote

$$
Z_{j}=\frac{\partial}{\partial z_{\jmath}}+i \frac{\partial f_{j}}{\partial z_{\jmath}} \frac{\partial}{\partial z_{n}} \quad \text { for } j=1, \ldots, n-1 .
$$

Then $\left\{Z_{1}, \ldots, Z_{n-1}\right\}$ is a basis of $T^{(1,0)}(\partial \Omega)$ near $\zeta$. Let $Z_{n}=N+i T$ a complex normal vector field, i.e., $Z_{n}$ is a $(1,0)$-vector field which is $\mathcal{C}^{\infty}(\bar{\Omega})$ and satisfies $Z_{n}(\rho)=1$ on $\partial \Omega$. It means that $Z_{n}$ goes outwards of $\Omega$, and $T=\partial / \partial t, t=\operatorname{Re}\left(z_{n}\right)$, is transverse to the complex tangent space at the boundary.

As in Nagel-Rosay-Stein-Wainger [NRSW] and Chang-Nagel-Stein [CNS], for every $\ell$-tuple of integers $\left(i_{1}, \ldots, i_{\ell}\right)$ we define smooth functions $\lambda_{i_{1}, \ldots, i_{\ell}}^{(j)}, \beta_{i_{1}, \ldots, i_{\ell}}^{(j)}$, and $\gamma_{i_{1}, \ldots, i_{\ell}}^{(j)}$, in a neighborhood of $\zeta$ by the equation

$$
\left[X_{i_{\ell}},\left[\cdots,\left[X_{i_{1}}, X_{i_{2}}\right] \cdots\right]\right]=\lambda_{i_{1}, \ldots, i_{\ell}}^{(j)} T+\beta_{i_{1}, \ldots, i_{\ell}}^{(j)} Z_{j}+\gamma_{i_{1}, \ldots, i_{\ell}}^{(j)} \bar{Z}_{j}
$$

with $X_{i_{\nu}} \in\left\{Z_{j}, \bar{Z}_{j}\right\}$ for $\nu=1, \ldots, \ell$ and $j=1, \ldots, n-1$.

For each integer $2 \leq \ell \leq m_{j}$, we define a smooth function $\Lambda_{j}^{(\ell)}$ in a neighborhood of $\zeta$ by the equation

$$
\Lambda_{j}^{(\ell)}(x)=\left[\sum_{\nu \leq \ell}\left|\lambda_{i_{1}, \ldots, i_{\nu}}^{(j)}(x)\right|^{2}\right]^{\frac{1}{2}} .
$$

Finally set

$$
\Lambda_{\jmath}(x, \delta)=\sum_{\ell=2}^{m_{j}} \Lambda_{j}^{(\ell)}(x) \delta^{\ell} .
$$

Let $h \rightarrow \mu_{j}(x, h)$ be the function inverse to $\delta \rightarrow \Lambda_{j}(x, \delta)$. Thus

$$
\mu_{j}(x, h) \approx \min _{2 \leq \ell \leq m_{\jmath}}\left(\frac{h}{\Lambda_{j}^{(\ell)}(x)}\right)^{\frac{1}{\ell}} \approx\left(\sum_{\ell=2}^{m_{J}}\left(\Lambda_{j}^{(\ell)}(x)\right)^{\frac{1}{\ell}} \cdot h^{-\frac{1}{\ell}}\right)^{-1}
$$

For $x, y \in \partial \Omega$ near $\zeta$, we may consider the distance $d(x, y)$ on $\partial \Omega$ (see Nagel-Stein-Wainger [NSW]) defined by:

$$
\begin{aligned}
d(x, y)=\min \{\delta>0: \Phi: & {[0,1] \rightarrow \partial \Omega, \Phi(0)=x, \Phi(1)=y, } \\
\text { such that } \Phi^{\prime}(t)= & \sum_{j=1}^{n-1}\left(a_{j}(t) Z_{j}+b_{j}(t) \bar{Z}_{j}\right)+c(t) T ; \\
& \left.\quad \text { with }\left|a_{j}(t)\right| \approx\left|b_{j}(t)\right| \leq \mu_{j}(x, \delta),|c(t)| \leq \delta\right\} .
\end{aligned}
$$


To obatin a globally defined pseudo-metric, we use a coordinate patch over $\partial \Omega$. This pseudo-metric is well defined and by the Campbell-Hausdorff formula, we can show that the corresponding balls $\mathcal{B}(x, \delta)$ are equivalent, up to a holomorphic change of coordinates, to a polydisc whose size is $\delta$ in the $T$ direction and $\mu_{j}(z, \delta)$ in the direction corresponding to $Z_{j}, j=1, \ldots, n-1$ (see McNeal [Mc2]). Furthermore, we have the following additional property on the functions $\mu_{j}$ 's which is that if $x \in \mathcal{B}(y, \delta)$ then $\mu_{j}(x, \delta) \simeq \mu_{j}(y, \delta)$.

The behavior of the Bergman kernel of finite type domains in $\mathbb{C}^{2}$ (see [Mc1], [NRSW]) as well as convex domains in $\mathbb{C}^{n}$ (see McNeal-Stein [McS], $[\mathrm{Mc} 3])$ are now well known. In this paper, we shall generalize results of Bonami-Grellier [BG] as well as Bonami-Chang-Grellier [BCG] about weighted Bergman projections on finite type domains in $\mathbb{C}^{2}$ to decoupled domains in $\mathbb{C}^{n}$.

It is well-known that the Bergman projection operator (even with respect to a smooth weight function) is smooth away from the diagonal $\Sigma=\{(x, y) \in \partial \Omega \times \partial \Omega: x=y\}$ on the boundary. Therefore, the corresponding Bergman kernel function and its derivatives are uniformly bounded on compact subsets of $\Omega$, we may concentrate the behavior of the kernel function near a neighborhood $U$ of $\partial \Omega$ in $\bar{\Omega}$ where the projection $\pi: U \rightarrow \partial \Omega$ is well-defined.

Let $d V$ denote the Lebesgue measure in $\mathbb{C}^{n}$, and let $d V_{\alpha}, \alpha \in \mathbb{Z}_{+}=$ $\{0,1,2, \ldots\}$, denote the weighted measure

$$
d V_{\alpha}(z)=\rho^{\alpha}(z) d V(z) .
$$

Let $\mathbf{B}_{\alpha}$ be the weighted Bergman projection for the measure $d V_{\alpha}$, i.e., the orthogonal projection in $L^{2}\left(d V_{\alpha}\right)$ onto $\mathcal{H}^{2}\left(d V_{\alpha}\right)$, the subspace of all $L^{2}\left(d V_{\alpha}\right)$ holomorphic functions. We use the notation $B_{\alpha}(z, w)$ to represent the corresponding weighted Bergman kernel, i.e.,

$$
\begin{aligned}
\mathbf{B}_{\alpha}(f)(z) & =\int_{\Omega} B_{\alpha}(z, w) f(w) d V_{\alpha}(w) \\
& =\int_{\Omega} B_{\alpha}(z, w) f(w) \rho^{\alpha}(w) d V(w) .
\end{aligned}
$$

For $z, w \in U$, we define

$$
D(z, w)=d(\pi(z), \pi(w))+\rho(z)+\rho(w) .
$$

Furthermore, for any $w \in U$, we define the "tent" over the ball

$$
\mathcal{B}(\pi(w), \delta)=\{z \in \partial \Omega, \quad d(z, \pi(w)) \leq \delta\}
$$


as the set

$$
\mathcal{B}^{\#}(w, \delta)=\bar{\Omega} \cap\left\{z \in \mathbb{C}^{n}, \quad D(z, w) \leq \delta\right\}
$$

Then

$$
\operatorname{Vol}_{\alpha}\left[\mathcal{B}^{\#}(w, \delta)\right] \simeq\left[\prod_{j=1}^{n-1} \mu_{j}^{2}(w, \delta)\right] \times \delta^{2+\alpha}
$$

The first step to understand the weighted Bergman projection is to establish the following size estimates for $B_{\alpha}(z, w)$ for $(z, w) \in \bar{\Omega} \times \bar{\Omega} \backslash \Sigma$.

TheOREm 1.1. Let $\alpha \in \mathbb{Z}_{+}$. For each $\ell$, there exists a constant $C_{\ell}$ so that if $Y_{1}, \ldots, Y_{\ell}$ are vector fields (acts either in $z$ or $w$ variable), each of which is one of $\left\{Z_{\jmath}, \bar{Z}_{\jmath}\right\}_{j=1}^{n}$, with $\beta_{k}$ either $Z_{k}$ or $\bar{Z}_{k}, k=1, \ldots, n-1$, and $\beta_{n}=\ell-\left(\beta_{1}+\cdots+\beta_{n-1}\right)$ either $Z_{n}$ or $\bar{Z}_{n}$, then

$$
\left|Y_{1} \ldots Y_{\ell} B_{\alpha}(z, w)\right| \leq C_{\ell} \frac{\left[\prod_{j=1}^{n-1} \mu_{j}^{-\beta_{j}}(z, D(z, w))\right] \times D^{-\beta_{n}}(z, w)}{\operatorname{Vol}_{\alpha}[\mathcal{B} \#(z, D(z, w))]}
$$

for any $(z, w) \in \bar{\Omega} \times \bar{\Omega} \backslash \Sigma$.

Next, we may use techniques in $[\mathrm{BCG}]$ to obtain the commutation relations with vector fields $T$ and $Z_{j}, j=1, \ldots, n-1$. From these commutation relations, it is easy to show Sobolev and Lipschitz estimates, both isotropic and anisotropic, for the operator $\mathbf{B}_{\alpha}$. Inspire of the work in $[\mathrm{McS}]$, we shall discuss $B M O\left(\Omega, d V_{\alpha}\right)$ estimate for the operator $\mathbf{B}_{\alpha}$ in the last section. The first author would like to thank Sandrine Grellier and Jeff McNeal for inspiring conversations about this project.

\section{$\S 2$. Operators of weighted Bergman type}

In order to obtain the commutation relations between the operator $\mathbf{B}_{\alpha}$ with vector fields $T$ and $Z_{j}, j=1, \ldots, n-1$, we need to introduce a class of operators called "operators of weighted Bergman type" and get some estimates for this class. For the convenience of our computation, let us fix the number $\alpha \in \mathbb{Z}_{+}$in the wieghted function $d V_{\alpha}$ in the rest of the paper.

Definition 2.1. A kernel $K(z, w) \in \mathcal{C}^{\infty}(\bar{\Omega} \times \bar{\Omega} \backslash \Sigma)$ corresponding to an operator $\mathbf{K}$ is a kernel of weighted Bergman type of order $\vec{r}=\left(r_{1}, \ldots, r_{n}\right)$ if it satisfies the following estimates:

For each $\ell$, there exists a constant $C_{\ell}$ so that if $Y_{1}, \ldots, Y_{\ell}$ are vector fields, each of which is one of $\left\{Z_{j}, \bar{Z}_{j}\right\}_{j=1}^{n}$, with $\beta_{k}$ either $Z_{k}$ or $\bar{Z}_{k}, k=$ 
$1, \ldots, n-1$, and $\beta_{n}=\ell-\left(\beta_{1}+\cdots+\beta_{n-1}\right)$ either $Z_{n}$ or $\bar{Z}_{n}$, then

$$
\left|Y_{1} \ldots Y_{\ell} K(z, w)\right| \leq C_{\ell} \frac{\left[\prod_{j=1}^{n-1} \mu_{j}^{r_{j}-\beta_{j}}(\pi(z), D(z, w))\right] \times D(z, w)^{r_{n}-\beta_{n}}}{\operatorname{Vol}_{\alpha}[\mathcal{B} \#(z, D(z, w))]}
$$

for $(z, w) \in \bar{\Omega} \times \bar{\Omega} \backslash \Sigma$. Each $Y_{\jmath}$ acts either in $z$ or $w$.

Remarks.

(1) Operators of weighted Bergman type of order $\overrightarrow{0}$ do not form an algebra. We need a further assumption on this class which is the action of $\mathbf{B}_{\alpha}$ on a suitable collection of bump functions. We shall discuss this point later. For more detail, see Stein [S, pp. 293-297].

(2) If $\mathbf{K}$ is a weighted Bergman type operator of order $\vec{r}=\left(r_{1}, \ldots, r_{n}\right)$, then from the definition, we know that $Z_{j} \mathbf{K}$ and $\bar{Z}_{\jmath} \mathbf{K}$ are weighted Bergman type operator of order $\left(r_{1}, \ldots, r_{j}-1, \ldots, r_{n}\right), j=1, \ldots, n$.

As we have mentioned in the above remark, we shall need to define a class of normalized "bump functions" as follows.

Definition 2.2. Let $M \in \mathbb{N}$. A function $\varphi$ of $\operatorname{class} \mathcal{C}^{M}$ in $\bar{\Omega}$ is said to be a bump function of order $M$ if $\varphi$ is supported in some $\mathcal{B}^{\#}(w, \delta)$ and if, for any $\ell \in \mathbb{N}, \ell \leq M$,

$$
\sup _{z \in \mathcal{B}^{\#}(w, \delta)}\left[\prod_{j=1}^{n-1} \mu_{j}^{\beta_{j}}(w, \delta)\right] \cdot \delta^{\beta_{n}} \cdot\left|Y_{1} \ldots Y_{\ell} \varphi(z)\right| \leq 1
$$

whenever $\beta_{k}$ of the $Y_{k}$ 's are either $Z_{k}$ or $\bar{Z}_{k}, k=1, \ldots, n-1$, and $\beta_{n}=$ $\ell-\left(\beta_{1}+\cdots+\beta_{n-1}\right)$ are either $Z_{n}$ or $\bar{Z}_{n}$.

Definition 2.3. We call a weighted Bergman type operator $\mathbf{K}$ of order $\vec{r}$ is "resticted regular" if both $\mathbf{K}$ and its adjoint $\mathbf{K}^{*}$ satisfy the following estimates:

For any $\ell \in \mathbb{N}$, there exists a constant $C_{\ell}$ and a positive integer $N_{\ell}$ such that, whenever $\varphi$ is a bump function of order $\geq N_{\ell}$ supported in $\mathcal{B}^{\#}(w, \delta)$, then

$$
\sup _{z \in \mathcal{B} \#(w, \delta)}\left|Y_{1} \ldots Y_{\ell} \mathbf{K}(\varphi)(z)\right| \leq C_{\ell}\left[\prod_{j=1}^{n-1} \mu_{\jmath}^{r_{\jmath}-\beta_{\jmath}}(w, \delta)\right] \times \delta^{r_{n}-\beta_{n}}
$$

whenever $\beta_{k}$ of the $Y_{k}$ 's are either $Z_{k}$ or $\bar{Z}_{k}, k=1, \ldots, n-1$, and $\beta_{n}=$ $\ell-\left(\beta_{1}+\cdots+\beta_{n-1}\right)$ are either $Z_{n}$ or $\bar{Z}_{n}$. 
From now on, we denote the class of all restricted regular weighted Bergman type operators of order $\vec{r}$ by $\mathcal{B}_{\vec{r}}^{(\alpha)}(\Omega)$. In fact, it can be shown that $\cup_{\vec{r} \in \mathbb{Z}^{n}} \mathcal{B}_{\vec{r}}^{(\alpha)}(\Omega)$ forms an algebra.

Remark. For the case $\Omega \subset \subset \mathbb{C}^{2}$ of finite type with smooth boundary, it has been proved independently in [Mc1] and in [NRSW] that the Bergman projection belongs to the class $\mathcal{B}_{\overrightarrow{0}}^{(\alpha)}(\Omega)$ with $\alpha=0$.

Define functions $h_{j}: \mathbb{R} \rightarrow\left\{2, m_{j}\right\}, j=1, \ldots, n-1$, as follows

$$
h_{j}(t)=2 \chi_{\{t \geq 0\}}+m_{j} \chi_{\{t<0\}}
$$

where $\chi_{E}$ is the characteristic function of the set $E$.

LEMma 2.4. There exists a constant $C$ such that, for any $\varepsilon>0$, any $\gamma_{1}, \ldots, \gamma_{n} \in \mathbb{R}$, and any $\zeta \in \partial \Omega$,

$$
\begin{aligned}
& \int_{0}^{\varepsilon} \prod_{j=1}^{n-1} \mu_{j}^{\gamma_{J}}(\zeta, t) \cdot t^{\gamma_{n}} d t \leq C \prod_{j=1}^{n-1} \mu_{j}^{\gamma_{J}}(\zeta, \varepsilon) \cdot \varepsilon^{\gamma_{n}+1} \\
& \text { if } \sum_{j=1}^{n-1} \frac{\gamma_{j}}{h_{j}\left(-\gamma_{j}\right)}+\gamma_{n}+1>0 \\
& \int_{\varepsilon}^{\infty} \prod_{j=1}^{n-1} \mu_{j}^{\gamma_{j}}(\zeta, t) \cdot t^{\gamma_{n}} d t \leq C \prod_{j=1}^{n-1} \mu_{j}^{\gamma_{J}}(\zeta, \varepsilon) \cdot \varepsilon^{\gamma_{n}+1} \\
& \text { if } \sum_{j=1}^{n-1} \frac{\gamma_{j}}{h_{j}\left(\gamma_{j}\right)}+\gamma_{n}+1<0 .
\end{aligned}
$$

Proof. By the definition of numbers $m_{j}$ and a basic property of functions $\mu_{j}(\zeta, \delta)$, we know that

$$
\left(\frac{\delta}{\delta^{\prime}}\right)^{\frac{1}{2}} \mu_{j}\left(\zeta, \delta^{\prime}\right) \leq \mu_{j}(\zeta, \delta) \leq\left(\frac{\delta}{\delta^{\prime}}\right)^{\frac{1}{m_{j}}} \mu_{j}\left(\zeta, \delta^{\prime}\right) .
$$

for all $z \in \partial \Omega$ and $0<\delta \leq \delta^{\prime} \leq \varepsilon$. Now we have

$$
\begin{aligned}
\int_{0}^{\varepsilon} \prod_{j=1}^{n-1} \mu_{j}(\zeta, t)^{\gamma_{j}} & \cdot t^{\gamma_{n}} d t \\
& =\sum_{k=1}^{\infty} \int_{2^{-k} \varepsilon}^{2^{-k+1} \varepsilon} \prod_{j=1}^{n-1} \mu_{j}^{\gamma_{j}}(\zeta, t) \cdot t^{\gamma_{n}} d t \\
& \leq C \cdot \sum_{k=1}^{\infty}\left[\prod_{j=1}^{n-1} \mu_{j}^{\gamma_{j}}\left(\zeta, 2^{-k} \varepsilon\right) \cdot\left(2^{-k} \varepsilon\right)^{\gamma_{n}+1}\right] \\
& \leq C \cdot\left(\prod_{j=1}^{n-1} \mu_{j}^{\gamma_{j}}(\zeta, \varepsilon) \cdot \varepsilon^{\gamma_{n}+1}\right) \cdot \sum_{k=1}^{\infty}\left(2^{-k}\right)^{\sum_{j=1}^{n-1} \frac{\gamma_{\jmath}}{h_{\jmath}\left(-\gamma_{\jmath}\right)}+\gamma_{n}+1}
\end{aligned}
$$


For the other integral, the computation is similar.

LEMMA 2.5. For any operator $\mathbf{K} \in \mathcal{B}_{\vec{r}}^{(\alpha)}(\Omega)$, there exists a constant $C_{\alpha}$ such that, for any $z \in \bar{\Omega}$, for any $\vec{\gamma}=\left(\gamma_{1}, \ldots, \gamma_{n}\right)$ with

$$
\sum_{j=1}^{n-1} \frac{r_{j}+\gamma_{j}}{h_{j}\left(-r_{j}-\gamma_{j}\right)}+r_{n}+\gamma_{n}+1>0,
$$

and

$$
\sum_{j=1}^{n-1} \frac{r_{j}+\gamma_{j}}{h_{j}\left(r_{j}+\gamma_{j}\right)}+r_{n}+\gamma_{n}<0
$$

such that the kernel $K(z, w)$ of $\mathbf{K}$ satisfying the following estimate:

$$
\begin{aligned}
\int_{\Omega}|K(z, w)| \times\left[\prod_{\jmath=1}^{n-1} \mu_{j}^{\gamma_{\jmath}}(\pi(w), \rho(w))\right] \times \rho^{\gamma_{n}}(w) d V_{\alpha}(w) \\
\leq C \cdot\left[\prod_{\jmath=1}^{n-1} \mu_{\jmath}^{\gamma_{\jmath}+r_{\jmath}}(\pi(z), \rho(z))\right] \times \rho^{\gamma_{n}+r_{n}}(z)
\end{aligned}
$$

Proof. We may rewrite the above integral as follows:

$$
\begin{aligned}
\int_{\Omega}|K(z, w)| \times\left[\prod_{j=1}^{n-1} \mu_{j}^{\gamma_{J}}(\pi(w), \rho(w))\right] \times \rho^{\gamma_{n}}(w) d V_{\alpha}(w) \\
=\int_{\mathcal{B}^{\#}(\pi(z), \rho(z))}+\sum_{k=0}^{\infty} \int_{\mathcal{B}^{\#}\left(\pi(z), 2^{k+1} \rho(z)\right) \backslash \mathcal{B}^{\#}\left(\pi(z), 2^{k} \rho(z)\right)}
\end{aligned}
$$

If $w \in \mathcal{B}^{\#}(\pi(z), \rho(z))$, then $\pi(w) \in \mathcal{B}(\pi(z), \rho(z))$. It follows that $\rho(w) \lesssim$ $\rho(z)$ and $\mu_{j}(\pi(w), \rho(w)) \approx \mu_{j}(\pi(z), \rho(w)) \lesssim \mu_{j}(\pi(z), \rho(z))$. Besides, we know that

$$
\rho(z) \leq D(z, w) \leq 3 \operatorname{rho}(z) .
$$

Therefore, the integral

$$
\begin{aligned}
\int_{\mathcal{B}^{\#}(\pi(z), \rho(z))}|K(z, w)| \times\left[\prod_{j=1}^{n-1} \mu_{j}^{\gamma_{j}}(\pi(w), \rho(w))\right] \times \rho^{\gamma_{n}}(w) d V_{\alpha}(w) \\
\leq C \cdot\left[\prod_{j=1}^{n-1} \mu_{j}^{\gamma_{\jmath}+r_{\jmath}}(\pi(z), \rho(z))\right] \times \rho^{\gamma_{n}+r_{n}}(z) .
\end{aligned}
$$


Similarly, we have

$$
\begin{aligned}
& \sum_{k=0}^{\infty} \int_{\mathcal{B}^{\#}\left(\pi(z), 2^{k+1} \rho(z)\right) \backslash \mathcal{B} \#\left(\pi(z), 2^{k} \rho(z)\right)} \\
&|K(z, w)| \prod_{j=1}^{n-1} \mu_{j}^{\gamma_{\jmath}}(\pi(w), \rho(w)) \times \rho^{\gamma_{n}}(w) d V_{\alpha}(w) \\
& \leq C_{\alpha} \cdot \sum_{k=0}^{\infty}\left(2^{k}\right)^{\sum_{\jmath=1}^{n-1} \frac{r_{\jmath}+\gamma_{\jmath}}{h_{j}\left(r_{\jmath}+\gamma_{\jmath}\right)}+r_{n}+\gamma_{n}}
\end{aligned}
$$

The above infinite series converges if $\sum_{j=1}^{n-1}\left(r_{j}+\gamma_{j}\right) /\left(h_{j}\left(r_{j}+\gamma_{j}\right)\right)+r_{n}+\gamma_{n}<$ 0 . This completes the proof of the lemma.

Corollary 2.6. Let $\alpha \in \mathbb{Z}_{+}$and let $\mathbf{K} \in \mathcal{B}_{\overrightarrow{0}}^{(\alpha)}(\Omega)$ be a restricted regular weighted Bergman type operator of order $\overrightarrow{0}$. Then the operator $\mathbf{K}$ orginally defined on $\mathcal{C}^{\infty}(\bar{\Omega})$ can be extended to a bounded operator from $L^{p}\left(d V_{\alpha}\right)$ to itself with $1<p<\infty$.

Proof. As usual (see [BG] and [BCG]), we may deduce this corollary by using Schur's Lemma (see Rudin $[\mathrm{R}]$ ). In fact, It suffices to find a positive function $g$ such that

$$
\int_{\Omega}|K(z, w)| g^{s}(w) d V_{\alpha}(w) \leq C \cdot g^{s}(z)
$$

where $s=p, p^{\prime}$. By Lemmas 2.4 and 2.5, the function $g=\rho^{-\varepsilon}$, with $\varepsilon$ small enough has this property $(0<\varepsilon<1)$. The proof of the corollary is therefore complete.

TheOREM 2.7. Let $\alpha \in \mathbb{N}$. Let $\mathbf{T}_{1}$ and $\mathbf{T}_{2}$ be two operators in $\mathcal{B}_{\vec{r}}^{(\alpha)}(\Omega)$ and in $\mathcal{B}_{\vec{s}}^{(\alpha)}(\Omega)$ respectively. Then $\mathbf{T}_{1} \mathbf{T}_{2}$ is in $\mathcal{B}_{\vec{r}+\vec{s}}^{(\alpha)}(\Omega)$ if the following condition holds:

$$
\nu=\sum_{j=1}^{n-1} \frac{r_{j}+s_{j}-2}{h_{j}\left(r_{j}+s_{j}-2\right)}+r_{n}+s_{n}-\alpha-2<0 .
$$

Proof. When $\alpha=0$, the proof can be reduced to composition of NIS operators in finite type domains in $\mathbb{C}^{2}$ (see [NRSW]) and decoupled domains in $\mathbb{C}^{n}$ (see Chang-Grellier [CG]). When $\alpha>0$, we borrow the same ideas from $[\mathrm{BG}]$ and $[\mathrm{BCG}]$. For completeness, we give some details of the proof 
here. It suffices to consider the operator $\mathbf{T}_{1} \mathbf{T}_{2}$, the conditions for its adjoint will follow.

First, we show that, under the assumption of the proposition, the kernel of $\mathbf{T}_{1} \mathbf{T}_{2}$ and its derivatives satisfy the right pointwise estimates. For $(z, w) \in \bar{\Omega} \times \bar{\Omega}$, let $\delta=D(z, w)$. Let $K_{1}$ and $K_{2}$ be the corresponding kernels of $\mathbf{T}_{1}$ and $\mathbf{T}_{2}$ respectively. We shall show that

$$
\left|K_{1} K_{2}(z, w)\right| \leq \frac{C \cdot\left[\sum_{j=1}^{n-1} \mu_{j}(z, \delta)^{r_{j}+s_{\jmath}}\right] \times \delta^{r_{n}+s_{n}}}{\operatorname{Vol}_{\alpha}\left[\mathcal{B}^{\#}(z, \delta)\right]} .
$$

The estimates for the derivatives will follow the same argument.

Let $\psi_{1}$ be some bump function supported in $\mathcal{B}^{\#}\left(z, \frac{\delta}{C}\right)$ and

$$
\psi_{1}(z)=1 \quad \text { on } \quad \mathcal{B}^{\#}\left(z, \frac{\delta}{2 C}\right)
$$

for some $C>1$ depending on the pseudo-distance $D$. We also assume that $\psi_{2}$ is a bump function supported in $\mathcal{B}^{\#}\left(w, \frac{\delta}{C}\right)$ and $\psi_{2}(z)=1$ in $\mathcal{B}^{\#}\left(w, \frac{\delta}{2 C}\right)$. Define

$$
\psi_{3}=1-\psi_{1}-\psi_{2}
$$

By definition

$$
K_{1} K_{2}(z, w)=\int_{\Omega} K_{1}(z, \zeta) K_{2}(\zeta, w) d V_{\alpha}(\zeta)
$$

So

$$
\begin{aligned}
K_{1} K_{2}(z, w)= & \int_{\Omega} K_{1}(z, \zeta) \\
& \quad+\int_{\Omega}(\zeta, w) \psi_{1}(\zeta) d V_{\alpha}(\zeta) \\
& \left.\quad+\int_{\Omega} K_{1}(z, \zeta) K_{2}(\zeta, w) \psi_{2}(\zeta) d V_{\alpha}(\zeta) w\right) \psi_{3}(\zeta) d V_{\alpha}(\zeta) \\
= & I_{1}+I_{2}+I_{3} .
\end{aligned}
$$

But

$$
\begin{aligned}
\left|I_{1}\right| & \leq\left|\mathbf{T}_{1}\left(\psi_{1}\right)(z)\right| \times \sup _{\zeta \in \operatorname{supp}\left(\psi_{1}\right)}\left|K_{2}(\zeta, w)\right| \\
& \leq\left|\mathbf{T}_{1}\left(\psi_{1}\right)(z)\right| \times \sup _{\zeta \in \operatorname{supp}\left(\psi_{1}\right)} \frac{\left[\sum_{j=1}^{n-1} \mu_{j}(w, D(\zeta, w))^{s_{\jmath}}\right] \times D(\zeta, w)^{s_{n}}}{\operatorname{Vol}_{\alpha}[\mathcal{B} \#(z, D(z, w))]} \\
& \leq \frac{C \cdot\left[\sum_{j=1}^{n-1} \mu_{j}(z, \delta)^{r_{j}+s_{\jmath}}\right] \times \delta^{r_{n}+s_{n}}}{\operatorname{Vol}_{\alpha}\left[\mathcal{B}^{\#}(z, \delta)\right]}
\end{aligned}
$$


since, on $\operatorname{supp}\left(\psi_{1}\right), D(\zeta, w) \simeq \delta$.

To handle $I_{2}$, the argument is similar (it uses the assumption on the adjoint of $\mathbf{T}_{2}$ ). It remains to estimate the last term $I_{3}$. Denote by $E$ the set of $\zeta \in \Omega$ where $D(z, \zeta) \geq \frac{\delta}{2 C}$ and $D(w, \zeta) \geq \frac{\delta}{2 C}$.

$$
\begin{aligned}
\left|I_{3}\right| & =\left|\int_{\Omega} K_{1}(z, \zeta) K_{2}(\zeta, w) \psi_{3}(\zeta) d V_{\alpha}(\zeta)\right| \\
& \leq C \int_{E}\left|K_{1}(z, \zeta) K_{2}(\zeta, w)\right| d V_{\alpha}(\zeta) \\
& \leq \int_{E \cap\{D(z, \zeta) \leq 2 \delta\}}+\int_{E \cap\{D(z, \zeta) \geq 2 \delta\}} \\
& =I_{3}^{(1)}+I_{3}^{(2)} .
\end{aligned}
$$

In the region of the integral $I_{3}^{(1)}$, we have $D(z, \zeta) \simeq \delta$ and $D(w, \zeta) \simeq \delta$. Therefore,

$$
\begin{aligned}
I_{3}^{(1)} & \leq \frac{C \cdot\left[\sum_{j=1}^{n-1} \mu_{j}(z, \delta)^{r_{\jmath}+s_{\jmath}}\right] \times \delta^{r_{n}+s_{n}}}{\left(\operatorname{Vol}_{\alpha}[\mathcal{B} \#(z, \delta)]\right)^{2}} \int_{D(z, \zeta) \leq 2 \delta} d V_{\alpha}(\zeta) \\
& \leq \frac{C \cdot\left[\sum_{j=1}^{n-1} \mu_{j}(z, \delta)^{r_{\jmath}+s_{j}}\right] \times \delta^{r_{n}+s_{n}}}{\operatorname{Vol}_{\alpha}[\mathcal{B} \#(z, \delta)]}
\end{aligned}
$$

In the region of the integral $I_{3}^{(2)}$, we have $D(\zeta, w) \simeq D(z, \zeta)$. Hence, by Lemma 2.5,

$$
\begin{aligned}
I_{3}^{(2)} & \leq C \int_{D(z, \zeta) \geq 2 \delta} \frac{\left[\sum_{j=1}^{n-1} \mu_{j}(z, \delta)^{r_{\jmath}+s_{\jmath}}\right] \times \delta^{r_{n}+s_{n}}}{\left(\operatorname{Vol}_{\alpha}[\mathcal{B} \#(z, D(z, \zeta)])^{2}\right.} d V_{\alpha}(\zeta) \\
& =\sum_{k=1}^{\infty} \int_{2^{k} \delta \leq D(z, \zeta)<2^{k+1} \delta} \frac{\left[\sum_{j=1}^{n-1} \mu_{j}(z, \delta)^{r_{j}+s_{j}}\right] \times \delta^{r_{n}+s_{n}}}{\left(\operatorname{Vol}_{\alpha}\left[\mathcal{B}^{\#}(z, D(z, \zeta)]\right)^{2}\right.} d V_{\alpha}(\zeta) \\
& \leq C \cdot \sum_{k=1}^{\infty} \frac{\left[\sum_{j=1}^{n-1} \mu_{j}(z, \delta)^{r_{j}+s_{\jmath}}\right] \times \delta^{r_{n}+s_{n}}}{\operatorname{Vol}_{\alpha}\left[\mathcal{B}^{\#}\left(z, 2^{k} \delta\right)\right]} \\
& \leq \frac{C \cdot\left[\sum_{j=1}^{n-1} \mu_{j}(z, \delta)^{r_{\jmath}+s_{\jmath}}\right] \times \delta^{r_{n}+s_{n}}}{\operatorname{Vol}_{\alpha}\left[\mathcal{B}^{\#}(z, \delta)\right]} \times \sum_{k=1}^{\infty}\left(2^{k}\right)^{\nu} .
\end{aligned}
$$

It remains to show that $\mathbf{T}_{1} \mathbf{T}_{2}$ is restrictedly regular. Let $\phi$ be a bump function supported in some $\mathcal{B}^{\#}(w, \delta)$ of order sufficiently large.

As in [NRSW], it is possible to construct a partition of unity $1=$ $\sum_{k \geq 0} \varphi_{k}$ with $\varphi_{k}$ a bump function supported in $\mathcal{B}^{\#}\left(w, c 2^{k} \delta\right) \backslash \mathcal{B}^{\#}\left(w, 2^{k} \delta\right)$, 
$k \geq 1$, where $c>1$ is some fixed constant, and $\varphi_{0}$ is a function supported in $\mathcal{B}^{\#}(w, c \delta)$.

We have

$$
\mathbf{T}_{2}(\phi): \sum_{k \geq 0} \varphi_{k} \mathbf{T}_{2}(\phi)
$$

But, using the properties of $\mathbf{T}_{2}$, we have

$$
\begin{aligned}
\varphi_{k} \mathbf{T}_{2}(\phi)(z) & =\varphi_{k}(z) \int_{\Omega} K_{2}(z, \zeta) \phi(\zeta) d V_{\alpha}(\zeta) \\
& =\gamma_{k} \phi_{k}(z)
\end{aligned}
$$

where $\phi_{k}$ is a bump function supported in $\mathcal{B}^{\#}\left(w, 2^{k} \delta\right)$ and

$$
\gamma_{k}=C \frac{\operatorname{Vol}_{\alpha}\left[\mathcal{B}^{\#}(w, \delta)\right]}{\operatorname{Vol}_{\alpha}\left[\mathcal{B}^{\#}\left(w, 2^{k} \delta\right)\right]} \times\left[\sum_{j=1}^{n-1} \mu_{j}\left(z, 2^{k} \delta\right)^{s_{\jmath}}\right] \times\left(2^{k} \delta\right)^{s_{n}} .
$$

For $k=0$, this follows immediately from the estimates on $\mathbf{T}_{2}(\phi)$. For $k \geq 1$, we use only the size estimates of the kernel $K_{2}(z, \zeta)$ and its derivatives which give (with the notations of Definition 2.1)

$$
\left|Y_{1} \ldots Y_{\ell} K_{2}(z, \zeta)\right| \leq \frac{c \cdot\left[\sum_{\jmath=1}^{n-1} \mu_{\jmath}\left(z, 2^{k} \delta\right)^{s_{j}-\beta_{\jmath}}\right] \times\left(2^{k} \delta\right)^{s_{n}-\beta_{n}}}{\operatorname{Vol}_{\alpha}\left[\mathcal{B}^{\#}\left(z, 2^{k} \delta\right)\right]}
$$

when $\zeta \in \mathcal{B}^{\#}(w, \delta)$ and $z \in \mathcal{B}^{\#}\left(w, c 2^{k} \delta\right) \backslash \mathcal{B}^{\#}\left(w, 2^{k} \delta\right)\left(\right.$ since $\left.D(z, \zeta) \simeq 2^{k} \delta\right)$. So

$$
\begin{aligned}
\mathbf{T}_{1} \mathbf{T}_{2} \phi(z) & =\int_{\Omega} K_{1}(z, \zeta) \mathbf{T}_{2}(\phi)(\zeta) d V_{\alpha}(\zeta) \\
& =\sum_{k \geq 0} \gamma_{k} \mathbf{T}_{1}\left(\phi_{k}\right)(z)
\end{aligned}
$$

so that

$$
\begin{aligned}
\sup _{z \in \mathcal{B}^{\#}(w, \delta)}\left|\mathbf{T}_{1} \mathbf{T}_{2}(\phi)(z)\right| & \leq \sum_{k \geq 0} \gamma_{k} \sup _{z \in \mathcal{B} \#(w, \delta)}\left|\mathbf{T}_{1}\left(\phi_{k}\right)(z)\right| \\
& \leq C \cdot\left\{\left[\sum_{j=1}^{n-1} \mu_{j}(w, \delta)^{r_{\jmath}+s_{\jmath}}\right] \times \delta^{r_{n}+s_{n}}\right\} \times \sum_{k=0}^{\infty}\left(2^{k}\right)^{\nu} \\
& \leq C\left[\sum_{j=1}^{n-1} \mu_{j}(w, \delta)^{r_{\jmath}+s_{j}}\right] \times \delta^{r_{n}+s_{n}} .
\end{aligned}
$$

The proof of the proposition is therefore complete. 
The next two results give simple rules of functional calculus in the operators of weighted Bergman type. The proofs of these two lemmas can be also founded in Bonami-Grellier [BG].

LEMma 2.8. Let $\alpha \in \mathbb{N}$ and let $\mathbf{K} \in \mathcal{B}_{\vec{r}}^{(\alpha)}(\Omega)$ be a restricted regular weighted Bergman type operator of order $\vec{r}$. Define the operator $\mathbf{K}^{\star}$ as follows:

$$
\mathbf{K}^{\star} f=\mathbf{K}(\rho f)
$$

Then $\mathbf{K}^{\star} \in \mathcal{B}_{\vec{r}+(0, \ldots, 0,1)}^{(\alpha+1)}(\Omega)$.

Proof. It is easy to see that the kernel $K^{\star}(z, w)$ of $\mathbf{K}^{\star}$ satisfies the right pointwise size estimates since $\operatorname{Vol}_{\alpha}\left[\mathcal{B}^{\#}(z, w)\right] \times D(z, w) \simeq \operatorname{Vol}_{(\alpha+1)}\left[\mathcal{B}^{\#}(z, w)\right]$. Next, we want to show that $\mathbf{K}^{\star}$ belongs to the class $\mathcal{B}_{\vec{r}+(0, \ldots, 0,1)}^{(\alpha+1)}(\Omega)$. Let $\varphi$ be any bump function supported in $\mathcal{B}^{\#}(w, \delta)$ of order sufficiently large. With the notations of Definition 2.3,

$$
\begin{aligned}
\sup _{z \in \mathcal{B} \#(w, \delta)}\left|Y_{1} \ldots Y_{\ell} \mathbf{K}^{\star}(\varphi)\right|(z) & =\sup _{z \in \mathcal{B} \#(w, \delta)}\left|Y_{1} \ldots Y_{\ell} \mathbf{K}(\rho \varphi)\right|(z) \\
& \leq C\left[\prod_{j=1}^{n-1} \mu_{j}(w, \delta)^{r_{\jmath}-\beta_{\jmath}}\right] \cdot \delta^{r_{n}+1-\beta_{n}}
\end{aligned}
$$

since $C \frac{\rho}{\delta} \varphi$ is a bump function. The same holds for the adjoint in $L^{2}\left(d V_{\alpha}\right)$ since the adjoint operator of $\mathbf{K}^{\star}$ equals to $\mathbf{K}^{*}(\rho \varphi)$.

Lemma 2.9. Let $\alpha \in \mathbb{N}$ and $\mathbf{K}$ be an operator in $\mathcal{B}_{\vec{r}}^{(\alpha)}(\Omega)$. Let

$$
\psi(z, w)=\prod_{j=1}^{n-1} \psi_{j}(z, w)
$$

be a $\mathcal{C}^{\infty}$-function in $\bar{\Omega} \times \bar{\Omega}$ satisfying

$$
\psi_{j}(z, w)=\mathcal{O}\left(\mu_{\jmath}^{\theta_{j}}(z, D(z, w))\right)
$$

for $\theta_{j} \in\{0,1\}, j=1, \ldots, n-1$. Let us consider the operator $\tilde{\mathbf{K}}$ defined by

$$
\tilde{\mathbf{K}} f(z)=T \mathbf{K}(\psi(z, \cdot) f)(z) .
$$

Then, $\tilde{\mathbf{K}} \in \mathcal{B}_{\left(\vec{r}+\left(\theta_{1}, \ldots, \theta_{n-1},-1\right)\right)}^{(\alpha)}(\Omega)$. 
Proof. It is obvious that the kernel of $\tilde{\mathbf{K}}$ is equal to

$$
\tilde{K}(z, w)=\left[T_{z} K(z, w)\right] \times\left[\prod_{\jmath=1}^{n-1} \psi_{j}(z, w)\right]
$$

and that it satisfies the right pointwise estimate. So is the kernel of its adjoint.

Let us consider the action of $\tilde{\mathbf{K}}$ on bump functions. Let $\varphi$ be any bump function supported in $\mathcal{B}^{\#}(w, \delta)$ of order sufficiently large.

$$
\begin{aligned}
\sup _{z \in \mathcal{B}^{\#}(w, \delta)}|\tilde{\mathbf{K}}(\varphi)|(z) & =\sup _{z \in \mathcal{B}^{\#}(w, \delta)}|T \mathbf{K}(\psi(z, \cdot) \varphi)|(z) \\
& \leq C \cdot\left[\prod_{j=1}^{n-1} \mu_{j}(w, \delta)^{r_{\jmath}+\theta_{\jmath}}\right] \cdot \delta^{r_{n}-1}
\end{aligned}
$$

since $\left[\prod_{j=1}^{n-1} \psi_{\jmath}(z, w) / \mu_{j}(z, \delta)^{\theta_{j}}\right] \cdot \varphi$ is bump for any $z \in \mathcal{B}^{\#}(w, \delta)$.

Next, let us consider the action of the adjoint operator of $\tilde{\mathbf{K}}$ on bump functions. Here we use the same bump function $\varphi$. Then, by integration by parts, we have

$$
\begin{aligned}
\tilde{\mathbf{K}}^{*}(\varphi(z)) & =\int_{\Omega}\left[T_{w} \bar{K}(w, z)\right] \bar{\psi}(w, z) \varphi(w) d V_{\alpha}(w) \\
& =\mathbf{K}^{*}\left(\bar{\psi}(\cdot, z) T \varphi+a_{\alpha}(z, \cdot) \varphi\right)(z)
\end{aligned}
$$

where $a_{\alpha}$ is a smooth function. Therefore, we have

$$
\begin{aligned}
& \sup _{z \in \mathcal{B}^{\#}(w, \delta)}\left|\tilde{\mathbf{K}}^{*}(\varphi)(z)\right| \leq C \cdot\left\{\left[\prod_{j=1}^{n-1} \mu_{j}(w, \delta)^{r_{\jmath}+\theta_{j}}\right] \cdot \delta^{r_{n}-1}\right. \\
&\left.\quad+\sum_{\jmath=1}^{n-1} \mu_{j}(w, \delta)^{r_{\jmath}}\left[\prod_{k \neq j} \mu_{k}(w, \delta)^{r_{k}+\theta_{k}}\right] \cdot \delta^{r_{n}}\right\} \\
& \leq C \cdot\left[\prod_{j=1}^{n-1} \mu_{\jmath}(w, \delta)^{r_{\jmath}+\theta_{\jmath}}\right] \cdot \delta^{r_{n}-1}
\end{aligned}
$$

since

$$
\left[\frac{\delta \cdot \bar{\psi}(\cdot, z)}{\prod_{j=1}^{n-1} \mu_{j}(w, \delta)^{\theta_{\jmath}}}\right] T \varphi
$$

is a bump function for any $z \in \mathcal{B}^{\#}(w, \delta)$ and $\delta \mu_{j}(w, \delta)^{-\theta_{\jmath}} \leq \delta^{1 / 2} \leq C$ for $j=1, \ldots, n-1$. The estimates for the derivatives are similar. The proof of this proposition is therefore complete. 


\section{$\S 3$. The weighted Bergman projection}

In the introduction, we have mentioned that the first step to obtain Sobolev and Lipschitz estimates for weighted Bergamn projections is to establish the size estimates. The following theorem will achieve this goal.

THEOREM 3.1. The weighted Bergman projection $\mathbf{B}_{\alpha}$ belongs to the class $\mathcal{B}_{\overrightarrow{0}}^{(\alpha)}(\Omega)$ for any $\alpha \in \mathbb{N}$.

Proof. We are going to prove this result by induction on $\alpha$. As we noticed before, the result is well known when $\alpha=0$ (see [BGC], [Mc1] and $[$ NRSW] $)$. Now, assume that $\mathbf{B}_{(\alpha-1)} \in \mathcal{B}_{\overrightarrow{0}}^{(\alpha-1)}(\Omega)$ for some $\alpha \geq 1$. We want to prove that $\mathbf{B}_{\alpha} \in \mathcal{B}_{\overrightarrow{0}}^{(\alpha)}(\Omega)$.

We first prove the following result by using the same method in [BG].

THEOREM 3.2. There exist an operator $\mathbf{H}_{\alpha} \in \mathcal{B}_{\overrightarrow{0}}^{(\alpha)}(\Omega)$ and two $\mathcal{C}^{\infty}$. functions $\Psi_{1}, \Psi_{2}$ in $\bar{\Omega} \times \bar{\Omega}$ such that

$$
\mathbf{B}_{\alpha}=\mathbf{H}_{\alpha}^{*}+\mathbf{E}_{\alpha} \mathbf{B}_{\alpha}=\mathbf{H}_{\alpha}-\mathbf{B}_{\alpha} \mathbf{E}_{\alpha} .
$$

with

$$
\mathbf{E}_{\alpha}=-i\left[\mathbf{B}_{\alpha-1} T\left(\Psi_{1} \rho f\right)-T \mathbf{B}_{\alpha-1}\left(\Psi_{1} \rho f\right)\right]
$$

an operator in the class $\mathcal{B}_{(1, \ldots, 1,0)}^{(\alpha)}(\Omega)$. Here $\mathbf{H}_{\alpha}^{*}$ is the adjoint of $\mathbf{H}_{\alpha}$ taken in $L^{2}\left(d V_{\alpha}\right)$.

Remark.

(1) From Theorem 3.2, it is easy to see

$$
\mathbf{B}_{\alpha}=\sum_{k=0}^{M}(-1)^{k} \mathbf{H}_{\alpha} \mathbf{E}_{\alpha}^{k}+(-1)^{M+1} \mathbf{B}_{\alpha} \mathbf{E}_{\alpha}^{M+1}
$$

for any $M \in \mathbb{N}$. This identity holds for general finite type domains in $\mathbb{C}^{2}$ as well as convex domains in $\mathbb{C}^{n}$, i.e., $\Omega$ is not necessary a domain like $\left\{\left(z_{1}, z_{2}\right): \operatorname{Im}\left(z_{2}\right)>f_{1}\left(z_{1}\right)\right\}$. When $\Omega$ is a decoupled domain, then we have $\mathbf{E}_{\alpha} \equiv 0$. This can be derived easily by using the special coordinates $\left(z_{1}, \ldots, z_{n-1}, t, \rho\right)$ on the neighborhood $U_{\zeta}$ and integration by parts. Here

$$
\rho=2 \operatorname{Im}\left(z_{n}\right)-\sum_{j=1}^{n-1} f_{j}\left(z_{j}\right), \quad t=\operatorname{Re}\left(z_{n}\right) .
$$


(2) The idea is to construct $\mathbf{H}_{\alpha}$ as a non-canonical projection in $L^{2}\left(d V_{\alpha}\right)$ to "approximate" the Bergman projection $\mathbf{B}_{\alpha}$. Such a construction has been first used in Kerzman-Stein [KS] for strictly pseudo-convex cases. However, the kernel for $\mathbf{H}_{\alpha}$ still relies on the kernel $\mathbf{B}_{\alpha-1}$. Therefore, we don't have a similar formula as [KS].

Proof of Theorem 3.2. First, since by assumption $\bar{Z}_{n} \rho=1$ on $\partial \Omega$, there exist two functions $\psi_{1}, \psi_{2}$ in $\mathcal{C}^{\infty}(\bar{\Omega}), \psi_{1} \equiv 1$ on $\partial \Omega$, so that

$$
1=\psi_{1}(w) \bar{Z}_{n} \rho(w)+\psi_{2}(w) \rho(w) .
$$

Let $f$ be a holomorphic function in $\mathcal{C}^{\infty}(\bar{\Omega})$. By the reproducing property, we have, for any $z \in \Omega$,

$$
\begin{aligned}
f(z)= & \int_{\Omega} B_{\alpha-1}(z, w) f(w) d V_{\alpha-1}(w) \\
= & \int_{\Omega} B_{\alpha-1}(z, w) f(w)\left[\psi_{1}(w) \bar{Z}_{n} \rho(w)+\psi_{2}(w) \rho(w)\right] d V_{\alpha-1}(w) \\
= & \int_{\Omega} B_{\alpha-1}(z, w) f(w) \psi_{1}(w) \frac{\bar{Z}_{n}(\rho(w))^{\alpha}}{\alpha} d V(w) \\
& \quad+\int_{\Omega} B_{\alpha-1}(z, w) f(w) \psi_{2}(w) \rho(w) d V_{\alpha-1}(w) .
\end{aligned}
$$

By integration by parts, there exists some smooth function $\psi_{3}$ such that

$$
f(z)=\int_{\Omega}\left[-\frac{\psi_{1}(w)}{\alpha} \bar{Z}_{n} B_{\alpha-1}(z, w)+\psi_{3}(w) B_{\alpha-1}(z, w)\right] f(w) d V_{\alpha}(w) .
$$

But $B_{\alpha-1}(z, w)$ is antiholomorphic in the $w$-variable, it follows that

$$
\bar{Z}_{n} B_{\alpha-1}(z, w)=-2 i T_{w} B_{\alpha-1}(z, w) .
$$

Therefore, we have

$$
f(z)=\int_{\Omega}\left[\frac{2 i}{\alpha} \psi_{1}(w) T_{w} B_{\alpha-1}(z, w)+\psi_{3}(w) B_{\alpha-1}(z, w)\right] f(w) d V_{\alpha}(w)
$$

This gives us two $\mathcal{C}^{\infty}(\bar{\Omega})$-functions $\Psi_{1}$ and $\Psi_{2}$ with $\Psi_{1}$ real valued on $\partial \Omega$, such that

$$
H_{\alpha}(z, w)=i \Psi_{1}(w) T_{w} B_{\alpha-1}(z, w)+\Psi_{2}(w) B_{\alpha-1}(z, w)
$$


is a reproducing kernel for the holomorphic functions in $\mathcal{C}^{\infty}(\bar{\Omega})$ with respect to the measure $d V_{\alpha}$. From a result of Ligocka [L], we know that $\mathbf{B}_{\alpha} f \in$ $\mathcal{C}^{\infty}(\bar{\Omega})$ if $f \in \mathcal{C}^{\infty}(\bar{\Omega})$, then we have

$$
\mathbf{B}_{\alpha} f=\mathbf{H}_{\alpha} \mathbf{B}_{\alpha} f
$$

On the other hand, we may use the identity

$$
\mathbf{H}_{\alpha} f=\mathbf{B}_{\alpha} \mathbf{H}_{\alpha} f
$$

to obtain $\mathbf{H}_{\alpha}^{*}\left(f-\mathbf{B}_{\alpha} f\right)=0$ since the operator $\mathbf{B}_{\alpha}$ is self-adjoint. Plugging in $(3.2)$, we get

$$
\begin{aligned}
\mathbf{B}_{\alpha} f & =\mathbf{H}_{\alpha}^{*} f+\mathbf{H}_{\alpha} \mathbf{B}_{\alpha} f-\mathbf{H}_{\alpha}^{*} f=\mathbf{H}_{\alpha}^{*} f+\mathbf{H}_{\alpha} \mathbf{B}_{\alpha} f-\mathbf{H}_{\alpha}^{*} \mathbf{B}_{\alpha} f \\
& =\mathbf{H}_{\alpha}^{*} f+\left(\mathbf{H}_{\alpha}-\mathbf{H}_{\alpha}^{*}\right) \mathbf{B}_{\alpha} f
\end{aligned}
$$

Therefore, we have

$$
\mathbf{B}_{\alpha} f=\mathbf{H}_{\alpha} f-\mathbf{B}_{\alpha}\left(\mathbf{H}_{\alpha}-\mathbf{H}_{\alpha}^{*}\right) f
$$

for $f \in \mathcal{C}^{\infty}(\bar{\Omega})$ which is dense in $L^{2}\left(d V_{\alpha}\right)$. Hence, (3.3) holds for $f \in$ $L^{2}\left(d V_{\alpha}\right)$.

It remains to show that $\mathbf{H}_{\alpha} \in \mathcal{B}_{\overrightarrow{0}}^{(\alpha)}(\Omega)$ and that

$$
\mathbf{E}_{\alpha}=\mathbf{H}_{\alpha}-\mathbf{H}_{\alpha}^{*} \in \mathcal{B}_{(1, \ldots, 1,0)}^{(\alpha)}(\Omega) .
$$

First, let us consider $\mathbf{H}_{\alpha}$. By construction, we know the kernel $H_{\alpha}^{*}(z, w)$ of the operator $\mathbf{H}_{\alpha}^{*}$ is

$$
H_{\alpha}^{*}(z, w)=-i \bar{\Psi}_{1}(z)\left[T_{z} B_{\alpha-1}\right](z, w)+\bar{\Psi}_{2}(z) B_{\alpha-1}(z, w) .
$$

It follows that

$$
\mathbf{H}_{\alpha}^{*}(f)(z)=-i \bar{\Psi}_{1}\left(T \mathbf{B}_{\alpha-1}\right)(\rho f)(z)+\bar{\Psi}_{2} \mathbf{B}_{\alpha-1}(\rho f)(z) .
$$

By assumption, $\mathbf{B}_{\alpha-1} \in \mathcal{B}_{\overrightarrow{0}}^{(\alpha-1)}(\Omega)$, then Lemmas 2.8 and 2.9 conclude that $\mathbf{H}_{\alpha} \in \mathcal{B}_{\overrightarrow{0}}^{(\alpha)}(\Omega)$.

Next, let us consider the operator $\mathbf{E}_{\alpha}$. We first look at the term corresponding to the kernel

$$
i \Psi_{1}(w) T_{w} B_{\alpha-1}(z, w)
$$


We have

$$
\begin{aligned}
i \int_{\Omega} T_{w} B_{\alpha-1} & (z, w) \Psi_{1}(w) f(w) d V_{\alpha}(w) \\
& =i \int_{\Omega} B_{\alpha-1}(z, w)\left[-T\left(\rho \Psi_{1} f\right)(w)+\Psi_{3}(w) \rho(w) f(w)\right] d V_{\alpha-1}(w) \\
& =(-i)\left[\mathbf{B}_{\alpha-1} T\left(\Psi_{1} \rho f\right)\right](z)+\left[\mathbf{B}_{\alpha-1}\left(\Psi_{3} \rho f\right)\right](z) .
\end{aligned}
$$

Hence,

$$
\begin{aligned}
\mathbf{E}_{\alpha}(f)(z)=\left[\mathbf { B } _ { \alpha - 1 } \left(\left(\Psi_{2}+\right.\right.\right. & \left.\left.\left.\Psi_{3}\right) \rho f\right)\right](z)-\bar{\Psi}_{2}(z)\left[\mathbf{B}_{\alpha-1}(\rho f)\right](z) \\
& -i\left[\mathbf{B}_{\alpha-1} T\left(\Psi_{1} \rho f\right)\right](z)+i \bar{\Psi}_{1}(z)\left[T \mathbf{B}_{\alpha-1}(\rho f)\right](z) \\
=\left[\mathbf { B } _ { \alpha - 1 } \left(\left(\Psi_{2}+\right.\right.\right. & \left.\left.\left.\Psi_{3}\right) \rho f\right)\right](z)-\bar{\Psi}_{2}(z)\left[\mathbf{B}_{\alpha-1}(\rho f)\right](z) \\
& -i\left[\mathbf{B}_{\alpha-1} T\left(\Psi_{1} \rho f\right)\right](z)+i\left[T \mathbf{B}_{\alpha-1}\left(\Psi_{1} \rho f\right)\right](z) \\
& -i\left[\Psi_{1}(z)-\bar{\Psi}_{1}(z)\right] T \mathbf{B}_{\alpha-1}(\rho f)(z) \\
& -i\left[T \mathbf{B}_{\alpha-1}\left(\left[\Psi_{1}-\Psi_{1}(z)\right] \rho f\right)\right](z) .
\end{aligned}
$$

Notice that

$$
\Psi_{1}(w)-\Psi_{1}(z)=\mathcal{O}\left(\prod_{\jmath=1}^{n-1} \mu_{j}(z, D(z, w))\right)
$$

and that, since $\Psi_{1}$ is real valued on $\partial \Omega$,

$$
\Psi_{1}(z)-\bar{\Psi}_{1}(z)=\mathcal{O}\left(\prod_{j=1}^{n-1} \mu_{j}(z, D(z, w))\right) .
$$

Therefore, by Lemmas 2.8 and 2.9, the corresponding operators of the last two terms are in $\mathcal{B}_{(1, \ldots, 1,0)}^{(\alpha)}(\Omega)$. Similarly, by Lemma 2.8 , the first two terms are in $\mathcal{B}_{(0, \ldots, 0,1)}^{(\alpha)}(\Omega)$, so in $\mathcal{B}_{(1, \ldots, 1,0)}^{(\alpha)}(\Omega)$.

It remains to prove that the remaining term,

$$
i\left\{\left[\mathbf{B}_{\alpha-1} T\left(\Psi_{1} \rho f\right)\right]-\left[T \mathbf{B}_{\alpha-1}\left(\Psi_{1} \rho f\right)\right]\right\}(z),
$$

is smoothing. In order to do that, we need the following lemma.

Lemma 3.3. There exist two $\mathcal{C}^{\infty}$-functions $\Phi_{1}$ and $\Phi_{2}$ in $\bar{\Omega} \times \bar{\Omega}$ so that

$$
\begin{aligned}
\mathbf{B}_{\alpha-1} T(\rho f)-T \mathbf{B}_{\alpha-1}(\rho f) & \\
& =\mathbf{B}_{\alpha-1}\left[\Phi_{2} \rho f\right]+\mathbf{B}_{\alpha-1}\left(\bar{\Phi}_{1} \mathbf{B}_{\alpha-1}(\rho f)\right)+\left[\mathbf{R}_{1}^{*}+\mathbf{R}_{1}\right](\rho f)
\end{aligned}
$$


where

$$
\mathbf{R}_{1} f(z)=\sum_{k=1}^{n}\left[\mathbf{I}-\mathbf{B}_{\alpha-1}\right]\left[\frac{\partial}{\partial z_{k}} \mathbf{B}_{\alpha-1}\right]\left(\phi_{k}(z, \cdot) \rho f\right)(z)
$$

with

$$
\phi_{j}(z, w)=\mathcal{O}\left(\prod_{j=1}^{n-1} \mu_{j}(z, D(z, w))\right)
$$

and $\mathbf{R}_{1}^{*}$ is the adjoint of $\mathbf{R}_{1}$ in $L^{2}\left(d V_{\alpha-1}\right)$.

Remark. We are going to show that the right hand side is smoothing. Close commutation properties can be found in the context of NIS operators (see $[\mathrm{CNS}]$ ).

Proof of Lemma 3.3. Let $\langle\cdot, \cdot\rangle$ be the inner product in the Hilbert space $L^{2}\left(d V_{\alpha-1}\right)$. Denote

$$
T_{z} B_{\alpha-1}(z, w)=\sum_{k=1}^{n} \gamma_{k}(z) \frac{\partial}{\partial z_{k}} B_{\alpha-1}(z, w)
$$

since $B_{\alpha-1}(z, w)$ is holomorphic in the $z$. For a $g \in L^{2}\left(d V_{\alpha-1}\right)$, we have

$$
\begin{aligned}
&\left\langle T \mathbf{B}_{\alpha-1}(\rho f)(z),\right.\left.g(z)-\left[\mathbf{B}_{\alpha-1} g\right](z)\right\rangle \\
&= \sum_{k=1}^{n}\left\langle\frac{\partial}{\partial z_{k}}\left[\mathbf{B}_{\alpha-1}\left(\gamma_{k}(z)-\gamma_{k}\right)(\rho f)\right](z), g(z)-\left[\mathbf{B}_{\alpha-1} g\right](z)\right\rangle \\
& \quad+\sum_{k=1}^{n}\left\langle\frac{\partial}{\partial z_{k}}\left[\mathbf{B}_{\alpha-1}\left(\gamma_{k} \rho f\right)\right](z), g(z)-\mathbf{B}_{\alpha-1} g(z)\right\rangle \\
&=\sum_{k=1}^{n}\left\langle\left[\mathbf{I}-\mathbf{B}_{\alpha-1}\right]\left\{\frac{\partial}{\partial z_{k}}\left[\mathbf{B}_{\alpha-1}\left(\left(\gamma_{k}(z)-\gamma_{k}\right) \rho f\right)\right]\right\}(z), g(z)\right\rangle \\
&=\left\langle\mathbf{R}_{1}(\rho f), g\right\rangle .
\end{aligned}
$$

Here we use the fact that

$$
\begin{aligned}
\sum_{k=1}^{n}\left\langle\frac{\partial}{\partial z_{k}}\left[\mathbf{B}_{\alpha-1}\left(\gamma_{k} \rho f\right)\right](z)\right. & \left., g(z)-\mathbf{B}_{\alpha-1} g(z)\right\rangle \\
& =\sum_{k=1}^{n} \frac{\partial}{\partial z_{k}}\left\langle\left[\mathbf{B}_{\alpha-1}\left(\gamma_{k} \rho f\right)\right](z), g(z)-\mathbf{B}_{\alpha-1} g(z)\right\rangle \\
& =\sum_{k=1}^{n} \frac{\partial}{\partial z_{k}}\left\langle\left[\mathbf{I}-\mathbf{B}_{\alpha-1}\right]\left[\mathbf{B}_{\alpha-1}\left(\gamma_{k} \rho f\right)\right](z), g(z)\right\rangle \\
& =\sum_{k=1}^{n} \frac{\partial}{\partial z_{k}}\left\langle\left[\left(\mathbf{B}_{\alpha-1}-\mathbf{B}_{\alpha-1}^{2}\right)\left(\gamma_{k} \rho f\right)\right](z), g(z)\right\rangle=0
\end{aligned}
$$


Similarly,

$$
\left\langle\left[\mathbf{I}-\mathbf{B}_{\alpha-1}\right](\rho f)(z), T \mathbf{B}_{\alpha-1}(g)(z)\right\rangle=\left\langle\mathbf{R}_{1}^{*}(\rho f), g\right\rangle .
$$

This gives us

$$
-\left\langle\mathbf{B}_{\alpha-1}(\rho f), T \mathbf{B}_{\alpha-1} g\right\rangle=-\left\langle\rho f, T \mathbf{B}_{\alpha-1} g\right\rangle+\left\langle\mathbf{R}_{1}^{*}(\rho f), g\right\rangle .
$$

Therefore, by integration by parts, we have

$$
\begin{aligned}
\left\langle T \mathbf{B}_{\alpha-1}(\rho f), g\right\rangle= & \left\langle T \mathbf{B}_{\alpha-1}(\rho f), \mathbf{B}_{\alpha-1} g\right\rangle+\left\langle\mathbf{R}_{1}(\rho f), g\right\rangle \\
=-\left\langle\mathbf{B}_{\alpha-1}(\rho f),\right. & \left.T \mathbf{B}_{\alpha-1} g\right\rangle \\
& \left\langle\mathbf{B}_{\alpha-1}(\rho f), \Phi_{1} \mathbf{B}_{\alpha-1} g\right\rangle+\left\langle\mathbf{R}_{1}(\rho f), g\right\rangle .
\end{aligned}
$$

Plugging (3.4) into the last line of (3.5), we have

$$
\begin{aligned}
\left\langle T \mathbf{B}_{\alpha-1}(\rho f), g\right\rangle=-\langle\rho f, & \left.T \mathbf{B}_{\alpha-1} g\right\rangle \\
& +\left\langle\left[\mathbf{R}_{1}^{*}+\mathbf{R}_{1}\right](\rho f), g\right\rangle+\left\langle\mathbf{B}_{\alpha-1}(\rho f), \Phi_{1} \mathbf{B}_{\alpha-1} g\right\rangle \\
=\left\langle T(\rho f), \mathbf{B}_{\alpha-1} g\right\rangle+\left\langle\Phi_{2} \rho f, \mathbf{B}_{\alpha-1} g\right\rangle & \\
\quad & \left\langle\left[\mathbf{R}_{1}^{*}+\mathbf{R}_{1}\right](\rho f), g\right\rangle+\left\langle\mathbf{B}_{\alpha-1}(\rho f), \Phi_{1} \mathbf{B}_{\alpha-1} g\right\rangle \\
=\left\langle\mathbf{B}_{\alpha-1} T(\rho f), g\right\rangle+\left\langle\mathbf{B}_{\alpha-1}\left(\Phi_{2} \rho f\right), g\right\rangle & +\left\langle\left[\mathbf{R}_{1}^{*}+\mathbf{R}_{1}\right](\rho f), g\right\rangle+\left\langle\mathbf{B}_{\alpha-1}\left(\Phi_{1} \mathbf{B}_{\alpha-1}(\rho f)\right), g\right\rangle .
\end{aligned}
$$

The last line of (3.6) holds because integration by parts. Hence we have

$$
\begin{aligned}
& T \mathbf{B}_{\alpha-1}(\rho f)-\mathbf{B}_{\alpha-1} T(\rho f) \\
& \quad=\mathbf{B}_{\alpha-1}\left(\Phi_{2} \rho f\right)+\mathbf{B}_{\alpha-1}\left(\bar{\Phi}_{1} \mathbf{B}_{\alpha-1}(\rho f)\right)+\left[\mathbf{R}_{1}^{*}+\mathbf{R}_{1}\right](\rho f) .
\end{aligned}
$$

Conclusion of the proof of Theorem 3.2. Now we may apply Lemma 3.3 to complete the proof of Theorem 3.2. By Lemmas 2.8 and 2.9, we get that the two first operators appearing in the preceding lemma are in $\mathcal{B}_{(0, \ldots, 0,1)}^{(\alpha)}(\Omega)$. It remains to consider $\mathbf{R}_{1}$. Let us prove that the operator defined by

$$
\tilde{\mathbf{K}}(f)(z)=\sum_{k=1}^{n} \frac{\partial}{\partial z_{k}} \mathbf{B}_{\alpha-1}\left(\phi_{k}(z, \cdot) \rho f\right)(z)
$$

is in $\mathcal{B}_{(1, \ldots, 1,0)}^{(\alpha)}(\Omega)$. It is first obvious that the kernels of $\tilde{K}$ and its adjoint satisfy the right estimates. Assume now that $\varphi$ is a bump function of order sufficiently large supported in some $\mathcal{B}^{\#}(w, \delta)$. It follows immediately from the definition of $\mathcal{B}_{\overrightarrow{0}}^{(\alpha-1)}(\Omega)$ that $\tilde{K}(\varphi)$ satisfies the right property. Let us 
consider the action of its adjoint. Since $\alpha \in \mathbb{N}$, then by integration by parts, we have

$$
\begin{aligned}
\tilde{\mathbf{K}}^{*}(\varphi)(z)= & \sum_{k=1}^{n} \int_{\Omega}\left[\frac{\partial}{\partial w_{k}} B_{\alpha-1}\right] \\
=\sum_{k=1}^{n} \int_{\Omega} B_{\alpha-1}(z, w) & {\left[\bar{\phi}_{k}(w, z) \frac{\partial \varphi}{\partial w_{k}}(w) \rho(w)\right] d V_{\alpha-1}(w) } \\
& +\int_{\Omega} B_{\alpha-1}(z, w)\left[B_{1}(z, w) \varphi(w) \bar{\phi}_{k}(w, z) d V_{\alpha}(w)\right. \\
& \left.+B_{2}(z, w) \varphi(w) \rho(w)\right] d V_{\alpha-1}(w) \\
= & \sum_{k=1}^{n} \mathbf{B}_{\alpha-1}\left[\bar{\phi}_{k}(\cdot, z) \frac{\partial \varphi}{\partial w_{k}} \rho+B_{1}(z, \cdot) \varphi \bar{\phi}_{k}(\cdot, z)+B_{2}(z, \cdot) \varphi \rho\right] .
\end{aligned}
$$

The result follows from the assumption on $\mathbf{B}_{\alpha-1}$ and from the fact that

$$
C \frac{\bar{\phi}_{k}(\cdot, z)}{\mu(w, \delta)} \rho \nabla \varphi
$$

is a bump function for any $z \in \mathcal{B}^{\#}(w, \delta)$.

Once we have Theorem 3.2, we may conclude the proof of Theorem 3.1 as follows.

Conclusion of the Proof of Theorem 3.1. By Theorem 3.2, we have

$$
\mathbf{B}_{\alpha}=\mathbf{H}_{\alpha}^{*}+\mathbf{E}_{\alpha} \mathbf{B}_{\alpha}
$$

So, by iteration,

$$
\mathbf{B}_{\alpha}=\sum_{k=0}^{M-1} \mathbf{E}_{\alpha}^{k} \mathbf{H}_{\alpha}^{*}+\mathbf{E}_{\alpha}^{M} \mathbf{B}_{\alpha} .
$$

Taking the adjoint in $L^{2}\left(d V_{\alpha}\right)$, we have also

$$
\mathbf{B}_{\alpha}=\sum_{k=0}^{M-1} \mathbf{H}_{\alpha}\left(\mathbf{E}_{\alpha}^{*}\right)^{k}+\mathbf{B}_{\alpha}\left(\mathbf{E}_{\alpha}^{*}\right)^{M} .
$$

By substitution in the above, we get

$$
\begin{aligned}
\mathbf{B}_{\alpha} & =\sum_{k=0}^{M-1} \mathbf{E}_{\alpha}^{k} \mathbf{H}_{\alpha}^{*}+\mathbf{E}_{\alpha}^{M} \sum_{k=0}^{M-1} \mathbf{H}_{\alpha}\left(\mathbf{E}_{\alpha}^{*}\right)^{k}+\mathbf{E}_{\alpha}^{M} \mathbf{B}_{\alpha}\left(\mathbf{E}_{\alpha}^{*}\right)^{M} \\
& =\mathbf{B}_{\alpha, M}+\mathbf{E}_{\alpha}^{M} \mathbf{B}_{\alpha}\left(\mathbf{E}_{\alpha}^{*}\right)^{M}
\end{aligned}
$$


where $\mathbf{B}_{\alpha, M}$ is, by Proposition 2.7, an operator in $\mathcal{B}_{\overrightarrow{0}}^{(\alpha)}(\Omega)$ for each $M$. Therefore it suffices to show that, for each fixed $\ell, M$ can be chosen large enough so that $Y_{1} \ldots Y_{\ell} E_{\alpha}^{M} B_{\alpha}\left(E_{\alpha}^{*}\right)^{M}(z, w)$ is bounded. This kernel corresponds to an operator of the form

$$
Y_{1} \ldots Y_{\ell_{1}} \mathbf{E}_{\alpha}^{M} \mathbf{B}_{\alpha}\left(\mathbf{E}_{\alpha}^{*}\right)^{M}\left(Y_{1} \ldots Y_{\ell_{2}}\right)^{*}
$$

for some $\ell_{1}, \ell_{2}$ so that $\ell_{1}+\ell_{2}=\ell$. So, it suffices to prove that for $M$ large enough, this operator sends $L^{1}\left(d V_{\alpha}\right)$ into $L^{\infty}$. Since $\mathbf{B}_{\alpha}$ maps $L^{2}\left(d V_{\alpha}\right)$ into itself, it remains to show that for $M$ large enough, $Y_{1} \ldots Y_{\ell_{1}} \mathbf{E}_{\alpha}^{M}$ maps $L^{2}\left(d V_{\alpha}\right)$ into $L^{\infty}$ and that $\left(\mathbf{E}_{\alpha}^{*}\right)^{M}\left(Y_{1} \ldots Y_{\ell_{2}}\right)^{*}$ maps $L^{1}\left(d V_{\alpha}\right)$ into $L^{2}\left(d V_{\alpha}\right)$. By Proposition 2.7, for $J$ large enough, we may assume that $Y_{1} \ldots Y_{\ell_{1}} \mathbf{E}_{\alpha}^{J}$ is in $\mathcal{B}_{\overrightarrow{0}}^{(\alpha)}(\Omega)$. So, we can see $Y_{1} \ldots Y_{\ell_{1}} \mathbf{E}_{\alpha}^{J}$ as a product of $M-J$ operators in $\mathcal{B}_{(1, \ldots, 1,0)}^{(\alpha)}(\Omega)$. Now, let $\mathbf{K}$ be an operator in $\mathcal{B}_{(1, \ldots, 1,0)}^{(\alpha)}(\Omega)$. From the pointwise estimates of the kernel of $\mathbf{K}$, it is easy to see that

$$
\int_{\Omega}|K(z, w)|^{r} d V_{\alpha}(w) \leq C
$$

independently of $z$ as long as

$$
1 \leq r \leq \frac{2 m+m \alpha+2}{2 m+m \alpha+1}
$$

Here $m=\max \left\{m_{1}, \ldots, m_{n-1}\right\}$ is the type of the domain $\Omega$. The same estimate holds for the adjoint of $\mathbf{K}$.

Hence such an operator maps $L^{1}\left(d V_{\alpha}\right)$ into $L^{r}\left(d V_{\alpha}\right)$ and $L^{r^{\prime}}\left(d V_{\alpha}\right)$ into $L^{\infty}$. By interpolation, we conclude that such an operator maps $L^{p_{1}}\left(d V_{\alpha}\right)$ into $L^{p_{2}}\left(d V_{\alpha}\right)$ if $1 / p_{2}=1 / p_{1}-1 / r^{\prime}$. This completes the proof of Theorem 3.1 .

Remarks.

(1) From Theorem 3.2, it is easy to see the following identity:

$$
B_{\alpha}(z, w)=D_{w} B_{\alpha-1}(z, w)+F_{\alpha}(z, w)
$$

where $\mathbf{F}_{\alpha} \in \mathcal{B}_{(1, \ldots, 1,0)}^{(\alpha)}(\Omega)$ is the operator $-\mathbf{B}_{\alpha} \mathbf{E}_{\alpha}$ and $D$ is a first order differential operator with $\mathcal{C}^{\infty}(\bar{\Omega})$-coefficients. Once again, if we use the coordinates $\left(z_{1}, \ldots, z_{n-1}, t, \rho\right)$ on a decoupled domain, we do have the exact identity $B_{\alpha}(z, w)=D_{w} B_{\alpha-1}(z, w)$. 
(2) Instead of $f$, we may consider $\mathbf{B}_{\alpha-1} f$ in (3.1). Then by the same integration by parts, we get

$$
\mathbf{B}_{\alpha-1} f(z)=\mathbf{H}_{\alpha} f(z)-\int_{\Omega} B_{\alpha-1}(z, w)[\bar{R} f(w)] \psi_{1}(w) d V_{\alpha}(w)
$$

if $f$ is not a holomorphic function. By Theorem 3.2, we have

$$
\mathbf{B}_{\alpha-1}=\mathbf{B}_{\alpha}-\mathbf{B}_{\alpha-1}\left(\rho \sum_{k=1}^{n} \bar{\gamma}_{k} \frac{\partial}{\partial \bar{z}_{k}}\right)-\mathbf{F}_{\alpha}
$$

\section{$\S 4$. Sobolev and Lipschitz estimates for weighted Bergman pro- jections}

The main object of this section is to prove the following:

$$
\mathbf{B}_{\alpha}: L_{k}^{p}\left(d V_{\alpha}\right) \rightarrow L_{k}^{p}\left(d V_{\alpha}\right),
$$

boundedly for $1<p<\infty$ and $k \in \mathbb{Z}_{+}$. In order to achieve this goal, we need the following result on commutation properties for $\mathbf{B}_{\alpha}$.

Theorem 4.1. Let $M N \alpha \in \mathbb{N}$.

(1) There exist operators $\left\{\mathbf{B}_{\alpha, i}\right\}_{l=0}^{M}$ in $\mathcal{B}_{\overrightarrow{0}}^{(\alpha)}(\Omega)$ such that

$$
\nabla^{M} \mathbf{B}_{\alpha}=\sum_{i=0}^{M} \mathbf{B}_{\alpha, i} T^{i}
$$

(2) Let $Z_{j}=\partial / \partial z_{j}+i \partial f_{j} / \partial z_{j} \partial / \partial z_{n}, j=1, \ldots, n-1$, be one of the complex tangential vector field on $\partial \Omega$. Then there exists $\left\{\mathbf{B}_{\alpha, i}\right\}_{i=0}^{N}$ operators in $\mathcal{B}_{(0, \ldots, N, \ldots, 0)}^{(\alpha)}(\Omega)$ such that

$$
\mathbf{B}_{\alpha}=\sum_{i=0}^{N} \mathbf{B}_{\alpha, i} Z_{j}^{i}
$$

(3) Let $\left\{Y_{i}\right\}_{i=0}^{M}$ be $M$ vector fields, each of which is $Z_{j}$ or $\bar{Z}_{j}, j=1, \ldots, n-$ 1 , then there exist operators $\left\{\mathbf{B}_{\alpha, i}\right\}_{\imath=0}^{M}$ in $\mathcal{B}_{(0, \ldots, M, \ldots, 0)}^{(\alpha)}(\Omega)$ such that

$$
Y_{1} \ldots Y_{M} \mathbf{B}_{\alpha}=\sum_{i=0}^{M} \mathbf{B}_{\alpha, i} Z_{j}^{i}
$$


Proof. Let $\vec{v}_{j}(\zeta)$ be the vector as follows

$$
\vec{v}_{\jmath}(\zeta)=\left[0, \ldots, 0, \stackrel{j}{1}, 0 \ldots, 0,-i \frac{\partial f_{j}}{\partial \zeta_{j}}(\zeta)\right] .
$$

We first observe that there exist two smooth functions $\eta_{1}$ and $\eta_{2}$ such that, for any $z, \zeta \in \bar{\Omega}$,

$$
1=\eta_{1}(z, \zeta)\left(Z_{j}\right)_{\zeta}^{i}\left(\left\langle\zeta-z, \vec{v}_{\jmath}(\zeta)\right\rangle^{i}\right)+\eta_{2}(z, \zeta)
$$

with $\eta(z, \zeta)=0$ in a neighborhood of the diagonal $\partial \Omega \times \partial \Omega$. Then we have

$$
\begin{aligned}
& \mathbf{B}_{\alpha}(f)(z) \\
& \quad=\int_{\Omega} B_{\alpha}(z, \zeta)\left[\eta_{1}(z, \zeta)\left(Z_{j}\right)_{\zeta}^{\imath}\left(\left\langle\zeta-z, \vec{v}_{\jmath}(\zeta)\right\rangle^{\imath}\right)+\eta_{2}(z, \zeta)\right] f(\zeta) d V_{\alpha}(\zeta),
\end{aligned}
$$

After $i$ integrations by parts, using the fact that $Z_{j}$ is tangent at the boundary and that $B_{\alpha}(z, \zeta)$ is antiholomorphic in $\zeta$, we obtain

$$
\begin{array}{rl}
\mathbf{B}_{\alpha}(f)(z)=\sum_{i=0}^{N} \int_{\Omega} B_{\alpha}(z, \zeta) \lambda_{i}(z, \zeta)\left(Z_{j}\right)_{\zeta}^{i} & f(\zeta) \cdot\left\langle\zeta-z, \vec{v}_{j}(\zeta)\right\rangle^{i} d V_{\alpha}(\zeta) \\
& +\int_{\Omega} B_{\alpha}(z, \zeta) \eta_{2}(z, \zeta) f(\zeta) d V_{\alpha}(\zeta)
\end{array}
$$

So, we conclude that

$$
\mathbf{B}_{\alpha}(f)(z)=\sum_{i=0}^{N} \mathbf{B}_{\alpha, i}\left(Z_{j}^{i} f\right)(z)
$$

where $\mathbf{B}_{\alpha, i}$ is the operator of kernel

$$
B_{\alpha, \imath}(z, \zeta)=B_{\alpha}(z, \zeta) \lambda_{i}(z, \zeta) \cdot\left\langle\zeta-z, \vec{v}_{j}(\zeta)\right\rangle^{i}
$$

for $i \geq 1$ and

$$
B_{\alpha, 0}(z, \zeta)=B_{\alpha}(z, \zeta) \lambda_{0}(z, \zeta) \cdot\left\langle\zeta-z, \vec{v}_{j}(\zeta)\right\rangle^{i}+B_{\alpha}(z, \zeta) \eta_{2}(z, \zeta) .
$$

The fact that $B_{\alpha, i}$, for $0 \leq i \leq N$, is of type $(0, \ldots, 0, \stackrel{\jmath}{i}, 0, \ldots, 0)$ follows from the estimate

$$
\left|\left\langle\zeta-z, \vec{v}_{j}(\zeta)\right\rangle\right| \leq C_{j} \mu_{j}(z, D(z, \zeta)) .
$$

This completes the proof of assertion (2). From (2) and the holomorphicity of $B_{\alpha}(z, \zeta)$ in the $z$-variable, conclusion (3) follows immediately. For (1), we refer to Theorem 4.1.1 in [BCG] where the proof for $\alpha=0$ is given. The general case needs a slight modification. We omit the details here. 
Before we state the main theorem of this section, we introduce some notations. Let $k$ be an integer. Let $L_{k}^{p}\left(d V_{\alpha}\right)$ be the usual Sobolev space related to the measure $d V_{\alpha}$. Similar to [CNS], let $N L_{k}^{p}\left(d V_{\alpha}\right)$ be the set of all $f \in L^{p}\left(d V_{\alpha}\right)$ so that

$$
\mathcal{P}(Z, \bar{Z}) f \in L^{p}\left(d V_{\alpha}\right)
$$

for all (noncommutative) polynomials $\mathcal{P}$ in $Z_{j}$ and $\bar{Z}_{j}, j=1, \ldots, n-1$, of degree at most $k$. To define the norm, let $\mathcal{A}_{k}$ be any (fixed) basis for the vector space of these polynomials and set

$$
\|f\|_{N L_{k}^{p}\left(d V_{\alpha}\right)}=\sum_{\mathcal{P} \in \mathcal{A}_{k}}\|\mathcal{P}(Z, \bar{Z}) f\|_{L^{p}\left(d V_{\alpha}\right)}
$$

For more detail of the spaces $N L_{k}^{p}\left(d V_{\alpha}\right)$, see Stein [S, pp. 605-610].

Define

$$
L_{k, T}^{p}\left(d V_{\alpha}\right)=\left\{f \in L^{p}\left(d V_{\alpha}\right) ; T^{i} f \in L^{p}\left(d V_{\alpha}\right), 1 \leq i \leq k\right\},
$$

and

$$
L_{k, Z_{\jmath}}^{p}\left(d V_{\alpha}\right)=\left\{f \in L^{p}\left(d V_{\alpha}\right) ; Z_{\jmath}^{i} f \in L^{p}\left(d V_{\alpha}\right), 1 \leq i \leq k\right\}
$$

Now the following theorem can be easily deduced from Theorem 4.1 and Corollary 2.6.

THEOREM 4.2. The weighted Bergman projection maps

$$
L_{k, T}^{p}\left(d V_{\alpha}\right) \rightarrow L_{k}^{p}\left(d V_{\alpha}\right)
$$

and

$$
L_{k, Z_{j}}^{p}\left(d V_{\alpha}\right) \rightarrow N L_{k}^{p}\left(d V_{\alpha}\right)
$$

for $1<p<\infty$ and $j=1, \ldots, n-1$. In particular, the isotropic and anisotropic spaces, $L_{k}^{p}\left(d V_{\alpha}\right)$ and $N L_{k}^{p}\left(d V_{\alpha}\right)$, are left invariant by $\mathbf{B}_{\alpha}$.

Next, let us consider Lipschitz estimates for $\mathbf{B}_{\alpha}$. We first introduce spaces $\Lambda_{T, \text { as }}^{\beta}(\Omega)$ as well as $\Lambda_{Z_{\jmath}, \text { as }}^{\beta}(\Omega), j=1, \ldots, n-1$ as follows. More precisely, for a function $g$ of one real variable let us define $\Delta_{h}^{k} g(t)$ as the $k$-th symmetric difference, which is obtained by induction from

$$
\Delta_{h} g(t)=g(t+h)-g(t)
$$


Let $\Phi_{T}=\Phi_{T}(\cdot, \zeta)$ be the integral curve of $T$ such that $\Phi_{T}(0)=\zeta$, and let $\Delta_{T, h}^{k} f(\zeta)=\Delta_{h}^{k}\left(f \circ \Phi_{T}\right)(0)$.

The space $\Lambda_{T, \text { as }}^{\beta}(\Omega)$ is the space of functions $f$ which are asymptotically Lipschitz in the $T$ direction. More precisely, let

$$
\Omega_{\varepsilon}=\{z \in \Omega ; \rho(z)<\varepsilon\},
$$

where $\rho$ denotes the distance to the boundary, and assume that, for $\zeta \in \Omega_{\varepsilon_{0}}$, $\Phi_{T}(\cdot, \zeta)$ is defined for $|t|<\varepsilon_{0}<\varepsilon$.

Definition 4.3. A function $f \in L^{\infty}(\Omega)$ belongs to $\Lambda_{T, \text { as }}^{\beta}(\Omega)$ if there exist an integer $k, k>\beta$ and a constant $C>0$, such that

$$
\left|\Delta_{h}^{\ell} f(\zeta)\right| \leq C|h|^{\ell-k}(\rho(\zeta)+|h|)^{\beta}, \quad \ell=0,1, \ldots, k
$$

for every $\zeta \in \Omega_{\varepsilon_{0}}$ and $|h|<\frac{\varepsilon_{0}}{k}$.

Similarly, we may define spaces $\Lambda_{Z_{\jmath} \text {,as }}^{\beta}(\Omega), j=1, \ldots, n-1$ as follows:

Definition 4.4. A function $f \in L^{\infty}(\Omega)$ belongs to $\Lambda_{Z_{3} \text {,as }}^{\beta}(\Omega)$ if the exist an integer $k, k>\beta$ and a constant $C>0$, such that

$$
\left|\Delta_{Z_{\jmath}, h}^{k} f(\zeta)\right|=\left|\Delta_{h}^{k}\left(f \circ \Phi_{Z_{\jmath}}\right)(0)\right| \leq C|h|^{\ell-k}(\rho(\zeta)+|h|)^{\beta},
$$

$\ell=0, \ldots, k$, for every $\zeta \in \Omega_{\varepsilon_{0}}$ and $|h|<\frac{\varepsilon_{0}}{k}$.

Remarks.

(1) It can be shown (see [BCG]) that a function $f \in L^{\infty}(\Omega)$ belongs to $\Lambda_{T, \text { as }}^{\beta}(\Omega)$ if and only if there exist an integer $k, k>\beta$ and a constant $C$ such that, for every $\ell \geq 0, f$ may be written as $b+g_{1}+g_{2}$, where $g_{2}$ is supported outside $\Omega_{\varepsilon_{0} / 2}, b$ and $g_{1}$ supported in $\Omega_{\varepsilon_{0}}$, and

$$
\begin{gathered}
|b(\zeta)| \leq C\left(\rho(\zeta)+2^{-\ell}\right)^{\beta} \\
\sum_{j=0}^{k}\left|T^{j} g_{1}(\zeta)\right| \leq C 2^{k \ell}\left(\rho(\zeta)+2^{-\ell}\right)^{\beta} .
\end{gathered}
$$

(2) Similar to (4.1), it can be shown that a function $f \in L^{\infty}(\Omega)$ belongs to $\Lambda_{Z_{j} \text {,as }}^{\beta}(\Omega)$ if and only if there exist an integer $k, k m>\beta$ and a constant 
$C$ such that, for every $\ell \geq 0, f$ may be written as $f=b+g_{1}+g_{2}$, where $g_{2}$ is supported outside $\Omega_{\varepsilon_{0} / 2}, b$ and $g_{1}$ supported in $\Omega_{\varepsilon_{0}}$ and:

$$
\begin{aligned}
|b(\zeta)| & \leq C\left(\rho(\zeta)+2^{-\ell}\right)^{\beta} \\
\left(\mu_{j}\left(\zeta, 2^{-\ell}\right)\right)^{k} \sum_{i=0}^{k}\left|Z_{j}^{i} g_{1}(\zeta)\right| & \leq C\left(\rho(\zeta)+2^{-\ell}\right)^{\beta} .
\end{aligned}
$$

Now we may write our theorem on Lipschitz estimates for the operator $\mathbf{B}_{\alpha}$.

TheOREM 4.5. Let $\alpha \in \mathbb{Z}_{+}$. Then weighted Bergman projection $\mathbf{B}_{\alpha}$ $\operatorname{maps} \Lambda_{T, \text { as }}^{\beta}(\Omega)$ and $\Lambda_{Z_{\jmath}, \text { as }}^{\beta}(\Omega), j=1, \ldots, n-1$, into $\Lambda^{\beta}(\Omega)$.

Proof. The proof of this theorem follows [BCG] where the case $\alpha=0$ is considered.

Part (1). The Bergman projection maps $\Lambda_{T, \text { as }}^{\beta}(\Omega)$ into $\Lambda^{\beta}(\Omega)$.

As $\mathbf{B}_{\alpha}(f)$ is holomorphic, we have to show that, for $z \in \Omega$,

$$
\left|\nabla^{k} \mathbf{B}_{\alpha}(f)(z)\right| \leq C \rho(z)^{\beta-k}
$$

for some $k>\beta$. Let $z \in \Omega$ be fixed, $\rho(z)<\varepsilon_{0}$, and let $f=b+g_{1}+g_{2}$, with $\rho(z) \sim 2^{-\ell}$. There is no problem for $\nabla^{k} \mathbf{B}_{\alpha}\left(g_{2}\right)$ since it is supoorted in a compact subset of $\Omega$. By assumption on $b$, we have

$$
\begin{aligned}
& \int\left|\nabla^{k} B_{\alpha}(z, \zeta)\right| \cdot \mid b(\zeta) \mid d V_{\alpha}(\zeta) \\
& \leq C \int\left|\nabla^{k} B_{\alpha}(z, \zeta)\right| \cdot\left(\rho(\zeta)+2^{-\ell}\right)^{\beta} d V_{\alpha}(\zeta) \leq C \rho(z)^{\beta-k}
\end{aligned}
$$

by Lemma 2.5 since $\beta<k$ and since the Bergman kernel $B_{\alpha}(z, w)$ belongs to the class $\mathcal{B}_{\overrightarrow{0}}^{(\alpha)}(\Omega)$.

So, we have to deal with the term $g_{1}$. Now, let $k_{0}$ be the integer involved in the definition of $\Lambda_{T, \text { as }}^{\beta}(\Omega)$. Assume $k \geq k_{0}$. By Theorem 4.1 (1), we can write:

$$
\nabla^{k}\left(\mathbf{B}_{\alpha} g_{1}\right)=\sum_{i=0}^{k_{0}} \mathbf{B}_{\alpha, i} T^{i} g_{1},
$$

where $\mathbf{B}_{\alpha, i}$ is of Bergman type $\left(k_{0}-k, 0\right)$. Then, by $(4.1)$, we have

$$
\begin{aligned}
\left|\nabla^{k} \mathbf{B}_{\alpha} g_{1}(z)\right| & \leq C \sum_{i=0}^{k_{0}} \int\left|B_{\alpha, i}(z, \zeta)\right| \cdot 2^{\ell k_{0}}\left(\rho(\zeta)^{\beta}+2^{-\ell \beta}\right) d V_{\alpha}(\zeta) \\
& \leq C \rho(z)^{\beta-k}
\end{aligned}
$$


by Lemma 2.5 applied to each $B_{\alpha, i}$ for $k>\beta-k_{0}$.

Part (2). The Bergman projection maps $\Lambda_{Z_{\jmath} \text {,as }}^{\beta}(\Omega), j=1, \ldots, n-1$, into $\Lambda^{\beta}(\Omega)$. Without of loss generality, we may assume $j=1$. Now we may fix variables $\left(z_{2}, \ldots, z_{n-1}\right)$. Then as before, it is enough to show that

$$
\left|Y_{1} \cdots Y_{k} \mathbf{B}_{\alpha}(f)(z)\right| \leq C \rho(z)^{\beta-\lambda}
$$

for some $\lambda>\beta$. Here $Y_{\imath}=T, Z_{1}$ or $\bar{Z}_{1}$. Take again $f=b+g_{1}+g_{2}$, with $\rho(z) \sim 2^{-\ell}$. Majorization for $g_{2}$ is obvious. For the term $b$, we can do as Part (1).

For the terms coming from $g_{1}$, we use Theorem 4.1 (2) to write

$$
Y_{1} \cdots Y_{k} \mathbf{B}_{\alpha} g_{1}=\sum_{i=0}^{k_{0}} Y_{1} \cdots Y_{k} \mathbf{B}_{\alpha, i} Z_{1}^{i} g_{1}
$$

where the $Y_{1} \cdots Y_{k} \mathbf{B}_{\alpha, i}$ 's are Bergman operators of type $\left(k_{0}, 0, \ldots, 0,-k\right)$ and $k_{0}>m \beta$. We choose $k>\beta+k_{0} / 2$ and by Lemma 2.5 again, we get

$$
\int\left|\nabla^{\lambda} B_{\alpha, i}(z, \zeta)\right| \cdot \rho(\zeta)^{\beta} \mu_{1}(\zeta, \rho(z))^{-k_{0}} d V_{\alpha}(\zeta) \leq C \rho(z)^{\beta-k}
$$

This gives the result by the assumption on $g_{1}$. By a result of Grellier $[\mathrm{G}]$, we know that $\mathbf{B}_{\alpha}(f) \in \Lambda_{T, \text { as }}^{\beta}(\Omega)$. Now we may use the same result to obtain $\mathbf{B}_{\alpha}(f) \in \Lambda_{Z_{\jmath} \text {,as }}^{\beta}(\Omega), j=1, \ldots, n-1$. The proof of the theorem is therefore complete.

Remarks. We may also consider the extremal cases for Lipschitz estimates. When $\beta$ tends to 0 , the space $\Lambda^{\beta}(\Omega) \cap \mathcal{H}(\Omega)$ can be replaced either by the Bloch space $\mathbb{B}(\Omega)$ or by the space $B M O A(\Omega)$. Let us recall the definitions:

A holomorphic function $F$ belongs to $\mathbb{B}(\Omega)$ if and only if

$$
\sup _{\zeta \in \Omega} \rho(\zeta)|\nabla F(\zeta)|<\infty
$$

a holomorphic function $F$ belongs to $B M O A(\Omega)$ if and only if $F$ is in $\mathcal{H}^{2}(\Omega)$, and its boundary values $F^{*}$ are in the space $B M O(\partial \Omega)$ which is defined in terms of the anisotropic distance $d$ :

$$
\sup _{\zeta, r} \frac{1}{\sigma(\mathcal{B}(\zeta, r))} \int_{\mathcal{B}(\zeta, r)}\left|F^{*}(z)-F_{\mathcal{B}(\zeta, r)}^{*}\right|^{2} d \sigma(z)<\infty
$$


here $\sigma$ denotes the Euclidean measure on $\partial \Omega$ and $F_{\mathcal{B}(\zeta, r)}^{*}$ denotes the meanvalue of $F$ on $\mathcal{B}(\zeta, r)$. Since boundary values of holomorphic functions involved in definitions of spaces $\mathbb{B}(\Omega)$ and $B M O A(\Omega)$, we just consider the measure $d V=d V_{0}$ in the extremal cases.

Let $\nu$ be a positive measure on $\Omega$. We shall say that $\nu$ is a Carleson measure (i.e., $\nu \in \mathcal{C}(\Omega)$ ) if there exists a constant $C$ such that $\nu(\Omega) \leq C$ and if, for any $\zeta \in \partial \Omega$, any $0<\varepsilon \leq \varepsilon_{0}$,

$$
\nu\left(\mathcal{B}^{\#}(\zeta, \varepsilon)\right) \leq C \sigma(\mathcal{B}(\zeta, \varepsilon)) .
$$

The smallest constant $C$ satisfying the preceding property is called the Carleson norm of $\nu$ and is denoted by $\|\nu\|_{e}$.

It can be shown that $F \in B M O A(\Omega)$ if and only if $\rho(z)|\nabla F|^{2} d V(z)$ is a Carleson measure (see Krantz $[\mathrm{Kr}]$ for strongly pseudo-convex domains in $\mathbb{C}^{n}$ and $[\mathrm{BCG}]$ for finite type domains in $\mathbb{C}^{2}$ ), i.e.,

$$
\int_{\mathcal{B} \#(\zeta, r)} \rho(z)|\nabla F|^{2} d V(z) \leq C \sigma(\mathcal{B}(\zeta, r))
$$

In fact, following the idea in [BCG], we have:

THEOREM 4.6.

(1) The Bergman projection $\mathbf{B}_{0}$ maps the space

$$
\left\{f \in L^{2}(\Omega) ; \sum_{j=1}^{n-1} \mu_{j}(\zeta, \rho(\zeta))\left[|f(\zeta)|+\left|Z_{j} f(\zeta)\right|\right] \leq C\right\}
$$

into the Bloch space $\mathbb{B}(\Omega)$.

(2) The Bergman projection $\mathbf{B}_{0}$ maps the space

$$
\left\{f \in L^{2}(\Omega) ; \sum_{j=1}^{n-1}\left(\mu_{j}(\zeta, \rho(\zeta))\right)^{2}\left[|f(\zeta)|^{2}+\left|Z_{j} f(\zeta)\right|^{2}\right] \frac{d V}{\rho(\zeta)} \in \mathcal{C}(\Omega)\right\}
$$

into $B M O A(\Omega)$.

\section{$\S 5 . \quad$ Estimates of weighted Bergman projections in $B M O\left(\Omega, d V_{\alpha}\right)$}

In this section, we will study the behavior of weighted Bergman projections on $L^{\infty}(\Omega)$. The main idea of this section should go back to [McS]. Let $Q(z, \varepsilon)$ denote the Euclidean cube in $\mathbb{C}^{n}$ centered at $z$ of diameter $\varepsilon$. 
Definition 5.1. A function $f$ belongs to the space $B M O\left(\Omega, d V_{\alpha}\right)$ if $f \in L_{\mathrm{loc}}^{1}\left(d V_{\alpha}\right)$ satisfying

$$
\sup _{Q \subset \Omega} \frac{1}{\operatorname{Vol}_{\alpha}[Q(w, \varepsilon)]} \int_{Q(w, \varepsilon)}\left|f(z)-f_{Q}\right| d V_{\alpha}(z)<\infty,
$$

where $f_{Q}$ denotes the average value of $f$ on $Q(w, \varepsilon)$, i.e.,

$$
f_{Q}=\frac{1}{\operatorname{Vol}_{\alpha}[Q(w, \varepsilon)]} \int_{Q(w, \varepsilon)} f(\xi) d V_{\alpha}(\xi) .
$$

We need the following estimate of the kernel $B_{\alpha}(z, w)$ for which we need to obtain our BMO result.

Lemma 5.2. Let $B_{\alpha}(z, w)$ be the kernel of the weighted Bergman projection on $\Omega$. There is a constant $C$, independent of $z \in \Omega$, such that

$$
\int_{\Omega}\left|\nabla_{z} B_{\alpha}(z, w)\right| d V_{\alpha}(w) \leq C \cdot \rho(z)^{-1} .
$$

Proof. Let $U$ be a local coordinate patch as we have mentioned in Section 1 and $z=\left(z_{1}, \ldots, z_{n}\right)$ be the coordinates of $z$. Since each component of the gradient $\nabla_{z}$ can be represented as

$$
\sum_{\jmath=1}^{n}\left[a_{j}^{(\ell)} Z_{j}^{z}+b_{j}^{(\ell)} \bar{Z}_{j}^{z}\right], \quad \ell=1, \ldots, n
$$

and antiholomorphic derivatives of the kernel $B_{\alpha}(z, w)$ vanish, it suffices to estimate each $\sum_{j=1}^{n} a_{j}^{(\ell)} Z_{j}$ applied to $B_{\alpha}(z, w)$. The pointwise estimate (1.2) of the kernel $B_{\alpha}(z, w)$ implies

$$
\begin{aligned}
\int_{\Omega} \mid\left[\sum_{j=1}^{n} a_{j}^{(\ell)} Z_{j}^{z}\right] & B_{\alpha}(z, w) \mid d V_{\alpha}(w) \\
\lesssim & \sum_{j=1}^{n-1} \int_{\Omega} \frac{\mu_{j}(z, D(z, w))^{-1}}{\operatorname{Vol}_{\alpha}\left[\mathcal{B}^{\#}(z, D(z, w))\right]} d V_{\alpha}(w) \\
& \quad+\int_{\Omega} \frac{D(z, w)^{-1}}{\operatorname{Vol}_{\alpha}\left[\mathcal{B}^{\#}(z, D(z, w))\right]} d V_{\alpha}(w)
\end{aligned}
$$

where

$$
D(z, w)=d(\pi(z), \pi(w))+\rho(z)+\rho(w) .
$$


As we have discussed in Section 1, we know that

$$
\begin{aligned}
\operatorname{Vol}_{\alpha}\left[\mathcal{B}^{\#}(z, D(z, w))\right] & \simeq \prod_{j=1}^{n-1} \mu_{k}(z, D(z, w))^{2} \times D(z, w)^{2+\alpha} \\
& \simeq \prod_{j=1}^{n-1}\left(\sum_{\ell=2}^{m_{\jmath}} \Lambda_{j}^{(\ell)}(z)^{\frac{2}{\ell}} D(z, w)^{-\frac{2}{\ell}}\right) \times D(z, w)^{2+\alpha}
\end{aligned}
$$

Using the same idea of [McS, Lemma 4], the integral (5.1) is bounded by $\rho(z)^{-1}$ and the conclusion of the lemma follows immediately.

Proposition 5.3. If $f \in C^{1}(\Omega)$ and

$$
|\nabla f(z)| \lesssim \rho(z)^{-1}
$$

then $f \in B M O\left(\Omega, d V_{\alpha}\right)$.

Proof. Let $Q(w, \varepsilon) \subset \Omega$ be a cube in $\Omega$ and consider an arbitrary $z \in Q(w, \varepsilon)$. Then, by mean value theorem,

$$
\begin{aligned}
|f(z)-f(w)| & \leq \int_{0}^{1}\left|\frac{d}{d t} f(w+t(z-w))\right| d t \\
& \leq C \cdot \int_{0}^{1} \rho(w+t(z-w))^{-1} \cdot|z-w| d t
\end{aligned}
$$

However, we know that

$$
\rho(w+t(z-w)) \geq \rho(w)-t|z-w|
$$

It follows that

$$
\begin{aligned}
|f(z)-f(w)| & \lesssim \int_{0}^{1} \frac{|z-w|}{\rho(w)-t|z-w|} d t \\
& \lesssim \log \frac{\rho(w)}{\rho(w)-|z-w|} .
\end{aligned}
$$

Therefore, it follows form (5.2) that

$$
\int_{Q(w, \varepsilon)}|f(z)-f(w)| d V_{\alpha}(z) \lesssim \operatorname{Vol}_{\alpha}[Q(w, \varepsilon)]
$$


Thus,

$$
\begin{aligned}
\int_{Q(w, \varepsilon)} \mid & f(z)-f_{Q} \mid d V_{\alpha}(z) \\
\leq & \int_{Q(w, \varepsilon)}|f(z)-f(w)| d V_{\alpha}(z)+\left|\int_{Q(w, \varepsilon)}\right| f(w)-f_{Q}\left|d V_{\alpha}(z)\right| \\
\leq & \operatorname{Vol}_{\alpha}[Q(w, \varepsilon)] \\
& \quad+\int_{Q(w, \varepsilon)} \frac{1}{\operatorname{Vol}_{\alpha}[Q(w, \varepsilon)]} \int_{Q(w, \varepsilon)}|f(\xi)-f(w)| d V_{\alpha}(z) d V_{\alpha}(\xi) \\
& \lesssim \operatorname{Vol}_{\alpha}[Q(w, \varepsilon)] .
\end{aligned}
$$

This completes the proof of the proposition.

Now Lemma 5.2 and Proposition 5.3 combine to give the following theorem.

THEOREM 5.4. Let $\mathbf{B}_{\alpha}$ be the weighted Bergman projection associated to $\Omega$ for the measure $d V_{\alpha}$ with $\alpha \in \mathbb{Z}_{+}$. Then $\mathbf{B}_{\alpha}$ maps $L^{\infty}(\Omega)$ to $B M O(\Omega$, $\left.d V_{\alpha}\right)$ boundedly.

\section{REFERENCES}

[BG] A. Bonami and S. Grellier, Werghted Bergman projections in domains of finite type in $\mathbb{C}^{2}$, Contemporary Math., 189 (1995), 65-80.

[BCG] A. Bonami, D. C. Chang and S. Grellier, Commutation Propertıes and Lipschitz estimates for the Bergman and Szegö projections, Math. Zeit., 223 (1996), 275-302.

[CNS] D. C. Chang, A. Nagel, and E. M. Stein, Estimates for the $\bar{\partial}-$ Neumann problem in pseudoconvex domains of fintte type in $\mathbb{C}^{n}$, Acta Math., 169 (1992), 153-228.

[CG] D. C. Chang and S. Grellier, Régularité de la projectıon de Szegö dans domannes découplés de type fini de $\mathbb{C}^{n}$, C. R. Acad. Sci. Paris, 315 (1992), 1365-1370.

[FR] F. Forelli and W. Rudin, Projections on spaces of holomorphic functions in balls, Indiana Univ. Math. J., 24 (1974), 593-602.

[FS] G. B. Folland and E. M. Stein, Estimates for the $\bar{\partial}_{b}$-complex and analysts on the Heisenberg group, Comm. Pure Appl. Math., 27 (1974), 429-522.

[G] S. Grellier, Behavior of holomorphic functions in complex tangential directıons in a domain of finite type in $\mathbb{C}^{n}$, Publicacions Matematiques, 36 (1992), 1-41.

[K1] J. J. Kohn, Boundares of complex manifolds, Proceedings, Conference on Complex Analysis, Minneapolis, 1964 (1965), 81-94.

[K2] J. J. Kohn, Boundary behavior of $\bar{\partial}$ on weakly pseudo-convex manifolds of dimension two, J. Differentail Geom., 6 (1972), 523-542. 
[Kr] S. G. Krantz, Geometric Analysis and Function Spaces, CBMS, regional Conf. Series in Math. \#81, AMS, 1993.

[KS] N. Kerzman and E. M. Stein, The Szegö kernel in terms of Cauchy-Fantappie kernels, Duke Math. J., 45 (1978), 197-224.

[L] E. Ligocka, Forelli-Rudin Constructıons and weighted Bergman projections, Studia Math., 94 (1989), 257-272.

[Mc1] J. McNeal, Boundary Behavior of the Bergman kernel function in $\mathbb{C}^{2}$, Duke Math. J., 58 (1989), 499-512.

[Mc2] J. McNeal, Estimates on the Bergman kernels of convex domarns, Advances in Math., 109 (1994), 108-139.

[Mc3] J. McNeal, Local geometry of decoupled pseudoconvex domains, Complex Analysis, Vieweg, 1992, pp. 223-230.

[McS] J. McNeal and E. M. Stein, Mapping properties of the Bergman projection on convex domains of finite type, Duke Math. J., 73 (1994), 177-199.

[NRSW] A. Nagel, J.-P. Rosay, E. M. Stein, and S. Wainger, Estimates for the Bergman and Szegö kernels in $\mathbb{C}^{2}$, Ann. Math., 129 (1989), 113-149.

[NSW] A. Nagel, E. M. Sien, and S. Wainger, Balls and metrics defined by vector fields I: Basic properties, Acta Math., 155 (1985), 103-147.

[R] W. Rudin, Function Theory in the Unit Ball of $\mathbb{C}^{n}$, Grund. der Math. \#241, Springer-Verlag, Berlin-New York, 1980.

[S] E. M. Stein, Harmonic Analysis: Real-Variable Methods, Orthogonality, and Oscillatory Integrals, Princeton University Press, Princeton, New Jersy, 1993.

\author{
Der-Chen Chang \\ Department of Mathematics, \\ University of Maryland, \\ College Park, MD 20742, USA \\ drc@math . umd . edu \\ Bao Qin Li \\ Department of Mathematics, \\ Florida International University, \\ University Park, Miami, FL 33199, USA \\ libaoqin@zeus.fiu.edu
}

